\title{
Near-IR spectra of red supergiants and giants
}

\section{Models with solar and with mixing-induced surface abundance ratios ${ }^{\star}$}

\author{
A. Lançon ${ }^{1}$, P. H. Hauschildt ${ }^{2}$, D. Ladjal ${ }^{1,3}$, and M. Mouhcine ${ }^{4}$ \\ 1 Observatoire Astronomique de Strasbourg, Université L. Pasteur \& CNRS (UMR 7550), Strasbourg, France \\ e-mail: lancon@astro.u-strasbg.fr \\ 2 Hamburger Sternwarte, Gojenbergsweg 112, 21029 Hamburg, Germany \\ 3 Institute of Astronomy, Katholieke Universiteit, Celestijnenlaan 200 B, 3001 Leuven, Belgium \\ 4 Astrophysics Research Institute, Liverpool John Moores University, Twelve Quays House, Egerton Wharf, Birkenhead, \\ CH41 1LD, UK
}

Received 14 June 2006 / Accepted 2 January 2007

\section{ABSTRACT}

\begin{abstract}
Context. It remains difficult to interpret the near-IR emission of young stellar populations. One main reason is our incomplete understanding of the spectra of luminous red stars.

Aims. This work provides a grid of theoretical spectra of red giant and supergiant stars, that extends through optical and nearIR wavelengths. For the first time, models are also provided with modified surface abundances of $\mathrm{C}, \mathrm{N}$ and $\mathrm{O}$, as a step towards accounting for the changes that occur due to convective dredge-up in red supergiants or may occur at earlier evolutionary stages in the case of rotation. The aims are (i) to assess how well current models reproduce observed spectra, in particular in the near-IR; (ii) to quantify the effects of the abundance changes on the spectra; and (iii) to determine how these changes affect estimates of fundamental stellar parameters.

Methods. Spectra are computed with the model atmosphere code PHOENIX and compared with a homogeneous set of observations. Although the empirical spectra have a resolution of only $\lambda / \Delta \lambda \sim 1000$, we emphasize that models must be calculated at high spectral resolution in order to reproduce the shapes of line blends and molecular bands.

Results. Giant star spectra of class III can be fitted extremely well at solar metallicity down to $\sim 3400 \mathrm{~K}$, where difficulties appear in the modelling of near-IR $\mathrm{H}_{2} \mathrm{O}$ and $\mathrm{TiO}$ absorption bands. Luminous giants of class II can be fitted well too, with modified surface abundances preferred in a minority of cases, possibly indicating mixing in excess of standard first dredge-up. Supergiant stars show a larger variety of near-IR spectra, and good fits are currently obtained for about one third of the observations only. Modified surface abundances help reproducing strong $\mathrm{CN}$ bands, but do not suffice to resolve the difficulties. The effect of the abundance changes on the estimated $T_{\text {eff }}$ depends on the wavelength range of observation and can amount several $100 \mathrm{~K}$.

Conclusions. While theoretical spectra for giant stars are becoming very satisfactory, red supergiants require further work. The model grid must be extended, in particular to larger micro-turbulent velocities. Some observed spectra may call for models with even lower gravities than explored here (and therefore probably stellar winds), and/or with more extreme abundances than predicted by standard non-rotating evolution models. Non-static atmospheres models should also be envisaged.
\end{abstract}

Key words. stars: fundamental parameters - stars: abundances - infrared: stars - stars: atmospheres

\section{Introduction}

Red supergiants and red giants are the most luminous stars in, respectively, star forming or old passive galaxies. Being cool, they are the dominant sources of near-IR light. In highly reddened starburst galaxies, the near-IR light from red supergiants is sometimes the only direct information available on the stellar populations. Models for their spectra are thus important, even though they are also particularly difficult to construct (rich molecular line spectrum, extended atmospheres). If they are successful in reproducing empirical spectra, it will be legitimate to use them instead of observed spectral libraries in future analyses of galaxies.

Recently, Levesque et al. (2005) have shown that up-todate models compare well with optical observations of red

* Selected theoretical spectra (see text) can be retrieved in FITS format at CDS via anonymous ftp to cdsarc.u-strasbg.fr (130.79.128.5), or via

http://cdsweb.u-strasbg.fr/cgi-bin/qcat?J/A+A/468/205 supergiants, and shown that this success helps in explaining the location of observed red supergiants in the HR-diagram. At nearIR wavelengths $(1-2.5 \mu \mathrm{m})$, the most prominent molecular features are those of $\mathrm{CO}$ and $\mathrm{CN}$. Their sensitivity to surface gravity and effective temperature $\left(T_{\text {eff }}\right)$ has been the basis of the 8-colour classification system of White \& Wing (1978), although they are also sensitive to other parameters (Tsuji 1976; McWilliam \& Lambert 1984; McGregor 1987; Bessell et al. 1991; Origlia et al. 1997). Strong CN bands are predicted for low gravity stars with temperatures around $4500 \mathrm{~K}$, and are indeed observed in local red supergiants (Lançon \& Wood 2000) and in extragalactic objects such as the bright star clusters of M 82 (Lançon et al. 2003). However, it had not been verified until now whether models are capable of reproducing the strengths of various $\mathrm{CN}$ and $\mathrm{CO}$ bands throughout the near-IR range simultaneously. Nor whether they can match optical and near-IR properties together.

An important aspect not accounted for in recent collections of model spectra for red supergiants is internal mixing. Standard stellar evolution predicts non-solar surface abundance 
ratios due to convective dredge-up in the red supergiant phase (Iben 1966; Maeder 1981). Early observations had pointed out the inadequacy of solar abundance ratios in individual cases (e.g. $\alpha$ Ori, Beer et al. 1972). More recently, both theory and observations showed that main sequence rotation or other processes are capable of mixing CNO-cycle products into the atmosphere even before the red supergiant phase is reached (Maeder \& Meynet 2001; Trundle \& Lennon 2005). In red supergiants, $\mathrm{He}$ and ${ }^{14} \mathrm{~N}$ surface abundances are typically enhanced while ${ }^{16} \mathrm{O}$ and ${ }^{12} \mathrm{C}$ abundances are reduced. Modified abundances of $\mathrm{C}, \mathrm{N}$ and $\mathrm{O}$ alter the relative strengths of the predominant molecules.

In this paper, we present recent PHOENIX models specifically computed to address some of the above points. The emphasis is on the effects of non-solar abundance ratios of $\mathrm{C}, \mathrm{N}$ and $\mathrm{O}$; a more complete study of other parameters (in particular micro-turbulent velocities) has been started and will be reported in a forthcoming paper. The model assumptions are described in Sect. 2 and the predicted colours and molecular features in Sect. 3. In Sects. 4 and 5, the models are compared with spectroscopic data covering wavelengths from 1 to $2.4 \mu \mathrm{m}$ or, for a subsample, from 0.51 to $2.4 \mu \mathrm{m}$. Giants of class III, luminous giants of class II and supergiants of class I are discussed successively. The discussion in Sect. 6 focuses on fundamental parameter determinations from spectra, including the effects of mass and surface abundances on $T_{\text {eff. }}$ A brief conclusion summarizes the results.

\section{PHOENIX models with solar and modified abundances}

\subsection{Summary of model ingredients}

The model atmospheres and synthetic spectra were computed with PHOENIX version 13.11.00B. The model setup is identical to that of Kučinskas et al. (2005). We recall only the most relevant details here. The models are computed in spherical symmetry. A mixing length to pressure scale height ratio of 2.0 is used for all models. Dust is allowed to form in the atmospheres but is assumed to immediately rain out of the photospheric layers; therefore, no dust opacities are used in the models shown here. This is an important assumption for cool models with large extensions. In addition, all models presented here have large enough gravities to not produce a radiatively driven wind and, therefore, winds are not included.

The model spectra were computed specifically for comparison with data that has a spectral resolving power of order 1000 , i.e. $\Delta \lambda \simeq 10 \AA$ at $1 \mu \mathrm{m}$. Nevertheless, we emphasize that the model spectra must be computed at high spectral resolution before smoothing, in order to sample individual absorption line profiles properly and to obtain low resolution spectra that resemble observations (Fig. 1). We used a wavelength sampling step of $0.1 \AA$ throughout. Using only half these points produces negligible changes at $\lambda>8000 \AA(0.1 \% \mathrm{rms})$, and small variations in the shapes of the strongest optical bands at $\lambda<8000 \AA$ ( $2 \% \mathrm{rms}$ ). The small sampling step used here is an important change with respect to previous collections of PHOENIX spectra, which were computed with an initial wavelength sampling step of $2 \AA$ (e.g. Kučinskas et al. 2005; and models included in the library of Martins et al. 2005).

The models discussed cover effective temperatures ranging from 2900 to $5900 \mathrm{~K}$ and gravities in the set $\log (g)=\{-1,-0.5,0,1,2\} \quad\left(\mathrm{cm} \mathrm{s}^{-2}\right)$. The micro-turbulent velocity is set to a constant value of $v_{\text {mic }}=2 \mathrm{~km} \mathrm{~s}^{-1}$, except in
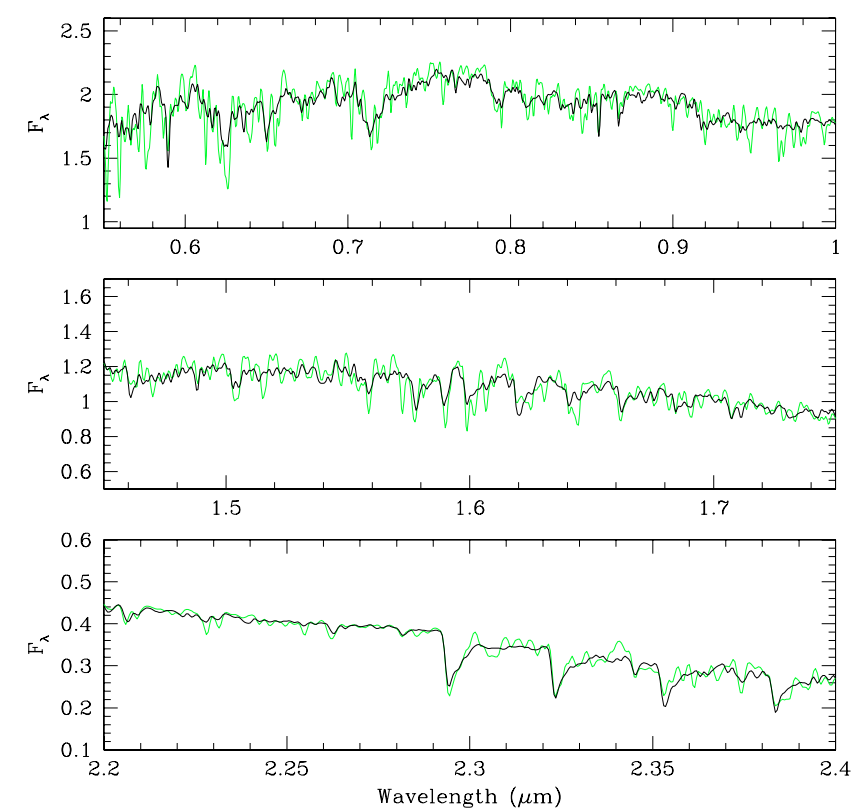

Fig. 1. Typical differences between PHOENIX spectra obtained with an initial wavelength sampling step of $2 \AA$ (grey) and $0.1 \AA$ (black). Both spectra have been smoothed by convolution with a Gaussian with $F W H M=15 \AA$. The models shown have $T_{\text {eff }}=4000 \mathrm{~K}, \log (g)=1$, $M=1 M_{\odot}$, but differences are important for any of the calculated models. Only the high resolution calculations match the data.

a few exploratory models. Values of 2 to $3 \mathrm{~km} \mathrm{~s}^{-1}$ are typical for red giant stars (Smith \& Lambert 1985). A more extensive grid of models covering higher values of this parameter is in the process of being calculated. Two stellar masses are considered: $1 M_{\odot}$ and $15 M_{\odot}$. Models at $9 M_{\odot}$ were also computed, but the differences with the $15 M_{\odot}$ ones are negligible. For $M=1 M_{\odot}$, many of the calculations at $\log (g)=-1$ did not converge (radiation pressure incompatible with the assumption of no winds), and this mass-gravity combination is therefore excluded from the discussion. We also restrict the discussion to optical and near-IR wavelengths, with a focus on wavelengths between 0.81 and $2.4 \mu \mathrm{m}$.

\subsection{Abundances}

The reference set of models assumes solar abundances, based on the review of Grevesse \& Noels (1993). The values most relevant to our study are summarized in Col. 2 of Table 1 . A subset of models with solar-scaled abundances but $\log \left(Z / Z_{\odot}\right)=-0.3$ was also computed but will only be discussed briefly in Sect. 6.2.

The second set of models has the same metallicity $Z=0.02$ as the reference set, but modified abundances of ${ }^{4} \mathrm{He},{ }^{12} \mathrm{C},{ }^{14} \mathrm{~N}$, ${ }^{16} \mathrm{O}$ (Col. 5 of Table 1 ). In the following, the adopted modified abundances will be refered to as "RSG-specific abundances".

The RSG-specific abundances were selected by inspection of the evolutionary tracks of Schaller et al. (1992; their case of standard mass loss) for stars with initial masses above $7 M_{\odot}$, at evolutionary timesteps with effective temperatures below $4500 \mathrm{~K}$. The values selected are representative of the final red supergiant stages of a star of initial mass $20 M_{\odot}\left(T_{\text {eff }} \simeq 3550 \mathrm{~K}\right)$. Stars of lower initial masses would have RSG abundances closer to the main sequence values, while the tracks at $25 M_{\odot}$ reach significantly larger modifications (tracks above $25 M_{\odot}$ don't extend to the low effective temperatures of red supergiants). Note that the initial mass fractions of Schaller et al. (1992) are not exactly 
Table 1. Surface abundances (by mass).

\begin{tabular}{c|lll|lll}
\hline \hline & Adopted & Geneva & Padova & Adopted & Geneva & Padova \\
& reference & 1992-1994 & 1993 & RSG-specific & 1994 & 1993 \\
$\begin{array}{c}\text { Element } \\
(1)\end{array}$ & set & ZAMS & ZAMS & set & RSG & RSG \\
\hline${ }^{1} \mathrm{H}$ & 0.703 & 0.680 & 0.700 & 0.580 & 0.55 & 0.63 \\
${ }^{4} \mathrm{He}$ & 0.280 & 0.300 & 0.280 & 0.400 & 0.43 & 0.35 \\
${ }^{12} \mathrm{C}$ & 0.0030 & 0.0044 & 0.0049 & 0.0022 & 0.0020 & 0.0032 \\
${ }^{14} \mathrm{~N}$ & 0.00092 & 0.0014 & 0.0012 & 0.0065 & 0.0080 & 0.0051 \\
${ }^{16} \mathrm{O}$ & 0.0083 & 0.0106 & 0.0106 & 0.0076 & 0.0068 & 0.0084 \\
\hline
\end{tabular}

Notes: Column 2: abundances adopted in our reference set of solar metallicity models. Column 3: for comparison, main sequence abundances of Schaller et al. (1992), also used by Meynet et al. (1994). Metal abundance ratios based on Anders \& Grevesse (1989). Column 4: main sequence abundances of Bressan et al. (1993). Metal abundance ratios based on Grevesse (1991). Column 5: abundances adopted in our RSG-specific set of models (see text). Column 6: final RSG abundances of Meynet et al. (1994) for $20 M_{\odot}$ stars. Column 7: final RSG abundances of Bressan et al. (1993) for $20 M_{\odot}$ stars.

the same as assumed in our reference set (mainly because of their larger He abundance), but that these differences are small compared to those that distinguish red supergiants from zero age main sequence stars.

In Table 1, the adopted abundances are compared to other values in the literature. The tracks of Meynet et al. (1994) assume larger mass loss rates than those of Schaller et al. (1992). More ingredients distinguish the models of Bressan et al. (1993) from those of the Geneva group. Nevertheless, predicted surface abundance alterations are of a comparable amplitude. Evolutionary tracks for stars with initial rotation predict that comparable ${ }^{14} \mathrm{~N}$ enhancements are reached already by the end of the main sequence (Meynet \& Maeder 2000, 2003), and are further increased during the RSG phase. The achieved abundance ratios depend strongly on initial rotation velocity, on initial mass and on mass loss prescriptions. One major difference between rotating and non-rotating models is the length of time a star spends as a red supergiant with modified abundances in one and the other case (see also Maeder \& Meynet 2001).

Mixing also occurs along the red giant branch and asymptotic giant branch for low and intermediate mass stars. The RSG-specific abundances adopted here are more extreme than those obtained through 1st dredge-up on the RGB (e.g. Iben 1964; Charbonnel et al. 1996; Girardi et al. 2000). In particular, the RSG-specific enrichment in $\mathrm{He}$ and ${ }^{14} \mathrm{~N}$ and the drop in $\mathrm{H}$ and ${ }^{12} \mathrm{C}$ are larger than expected from 1st dredgeup. More mixing may however occur through second dredgeup on the early asymptotic giant branch (for stars with initial masses above about $4 M_{\odot}$, e.g. Becker $\&$ Iben 1979; Boothroyd \& Sackmann 1999), and though "non-standard" extra mixing for low mass stars that have evolved on the red giant branch past the RGB-bump (Charbonnel 1994; Charbonnel \& do Nascimento 1998). Both these processes affect relatively luminous giant stars. The second one seems to be less efficient at the quasi-solar metallicities we consider than for population II stars. Therefore, we expect our RSG-specific abundances not to be appropriate for most solar neighbourhood giants of class III, while they might be relevant to some giants of class II.

We note that future calculations with modified surface abundances will include mixing-induced enhancements in the ${ }^{13} \mathrm{C}$ abundance, since ${ }^{13} \mathrm{CO}$ is a clear feature in near-IR spectra of cool stars. The effects of recent changes in measurements of the solar abundance ratios (Asplund et al. 2005) will also be investigated.

\subsection{Spectra in numerical form}

The model spectra for $M=1 M_{\odot}$ with solar abundances, for $M=15 M_{\odot}$ with solar abundances, and for $M=15 M_{\odot}$ with RSG-specific abundances, are made available in FITS format through CDS. Because the quality assessments made in this paper are restricted to resolutions of order $10^{3}$ in the near-IR (and a few hundred at wavelengths below $0.81 \mu \mathrm{m}$ ), the spectra made available are smoothed with a Gaussian to a full width at half maximum of $2 \AA$. The initial models, calculated with a wavelength step of $0.1 \AA$, can be requested from A.L. or P.H.H.

\section{Trends in the models}

Spectra illustrating the effects of mass, gravity and surface abundances are provided in Fig. 2. In this section, we will discuss quantitative trends using selected colours and molecular band indices. The indices are measured for each spectrum using: (i) the standard $J, H, K$ filter passbands of Bessell \& Brett (1988); (ii) narrow and intermediate-band filters as described in Table 2. All narrow and intermediate filter passbands are approximated with rectangles of identical central wavelength and width as the filters in the original references (as already done by Bessell et al. 1989). A model Vega spectrum provides the zero points in all passbands.

\subsection{Colours}

As shown in Fig. 3, colours that combine flux measurements around $1.04 \mu \mathrm{m}$, in the $J$ band and in the $K$ band are good indicators of $T_{\text {eff }}$ in theory, as their sensitivity to surface gravity is low. Above $3400 \mathrm{~K}$, the spread in $\log (g)$ corresponds to a full spread in $T_{\text {eff }}$ of about $200 \mathrm{~K}$ for the $15 M_{\odot}$ models (left panel). For $1 M_{\odot}$ models, the corresponding spread is much smaller: about $60 \mathrm{~K}$, centered on a line very close to the models at $15 M_{\odot}$ and $\log (g)=0$. At the lowest temperatures, contamination of the pseudo-continuum in the $K$ band with $\mathrm{H}_{2} \mathrm{O}$ absorption leads to reduced fluxes in low gravity stars. Unfortunately, in the twocolour plots useful for observers the extinction vectors run almost exactly parallel to the temperature sequence (right panel) : more resolved spectral information is necessary to estimate an effective temperature from near-IR data.

Figure 4 illustrates the gravity dependence of colours involving $H$ band fluxes. At high gravities, the minimum of the opacity of $H^{-}$around $1.6 \mu \mathrm{m}$ produces a distinct hump in the $H$ band spectra, with correspondingly blue $H-K$ and red $J-H$ colours. At low gravities, molecular absorption due mainly to $\mathrm{CO}$ and 

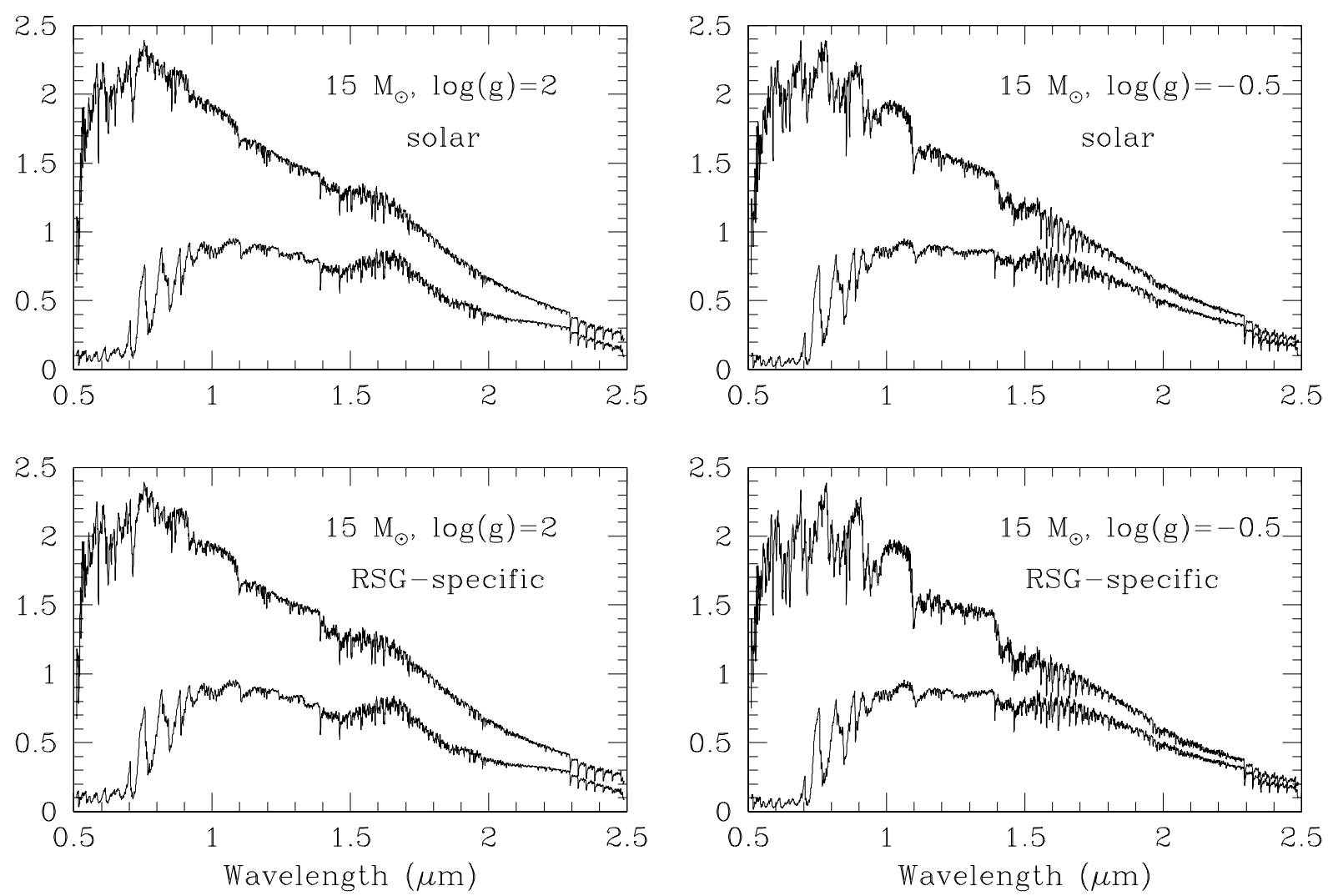

Fig. 2. Effects of gravity, temperature and surface abundances on PHOENIX model spectra. In each figure, the upper spectrum has $T_{\text {eff }}=4000 \mathrm{~K}$ and the lower one $T_{\mathrm{eff}}=3300 \mathrm{~K}$. For easier comparison, fluxes have been normalized to values comparable to those in the upper left diagram. The figures on the left are at $\log (g)=2$, those on the right at $\log (g)=-0.5$. The upper figures are for solar abundances, the lower ones for RSG-specific abundances. The effect of mass $\left(1 M_{\odot}\right.$ vs. $\left.15 M_{\odot}\right)$ is too small to be easily identified on this type of figure.

Table 2. Filter and index definitions.

\begin{tabular}{llll}
\hline \hline Filter & $\begin{array}{l}\text { Center } \\
(\mu \mathrm{m})\end{array}$ & $\begin{array}{l}\text { Width } \\
(\AA)\end{array}$ & Notes \\
\hline 104 & 1.0395 & 50 & quasi-continuum (1) \\
108 & 1.0800 & 60 & $\mathrm{CN}(1)$ \\
110 & 1.1000 & 50 & quasi-continuum near CN (1) \\
220 & 2.2000 & 1100 & quasi-continuum $(2)$ \\
236 & 2.3600 & 800 & 1st overtone CO (2) \\
$\mathrm{COH}$ & 1.6222 & 80 & 2nd overtone CO \\
$\mathrm{COHc} 1$ & 1.6160 & 30 & absorption-minimum near CO \\
$\mathrm{COHc} 2$ & 1.6285 & 30 & absorption-minimum near CO \\
\hline Index & Definition $(3)$ & \\
\hline $104-220$ & $-2.5 \log (104 / 220)+\mathrm{cst}$ \\
$\mathrm{CO}(2.3)$ & $-2.5 \log (236 / 220)+\mathrm{cst}$ \\
$\mathrm{CO}(1.6)$ & $-2.5 \log [2 \mathrm{COH} /(\mathrm{COHc} 1+\mathrm{COHc} 2)]+\mathrm{cst}$ \\
$\mathrm{CN}(1.1)$ & $-2.5 \log (110 / 108)+\mathrm{cst}$ \\
\hline
\end{tabular}

Notes: (1) Adapted from the 8-colour system of R.F. Wing (White \& Wing 1978). (2) Adapted from Frogel et al. (1978). (3) cst stands for a constant that gives the index the value 0 for a model spectrum of Vega.

$\mathrm{CN}$ erases this continuum opacity feature. Such an effect was already mentioned by Bessell et al. (1991), though their interpretation probably underestimated the rôle of $\mathrm{CN}$ as compared to $\mathrm{CO}$. The observations of Lançon et al. (2007) and those described in Kučinskas et al. (2005) provide a convincing validation of the $J H K$ colours of the new models.
The effect of mass on the $H$ band flux is insignificant at $\log (g)>0$. For lower gravities, $H-K$ increases by up to only 0.02 mag when going from 15 to $1 M_{\odot}$, at $T_{\text {eff }}>4000 \mathrm{~K}$.

Switching from solar-scaled to RSG-specific abundances has the following (small) effects on the above colours. All colours tend to become bluer. Colour differences in $H-K$ and $J-H$ remain smaller than $0.04 \mathrm{mag}$ (and are $<0.02$ mag for most stellar parameters). The colour index 104-220 (defined in Table 2) is reduced by up to 0.08 mag. The bolometric corrections to the $K$ band, BC(K), are essentially unchanged (the PHOENIX values agree with those of Levesque et al. 2005, to within a few percent between 3400 and $4300 \mathrm{~K}$ ). Effects this small would be difficult to exploit on real stellar spectra.

\subsection{Molecular indices}

\subsubsection{CO}

$\mathrm{CO}$ is a long known indicator of luminosity class (Baldwin et al. 1973). It is sensitive to gravity and effective temperature, but also to metallicity and micro-turbulence. As indicated previously, a constant micro-turbulent velocity of $2 \mathrm{~km} \mathrm{~s}^{-1}$ is used in this paper except in a few models. Large micro-turbulent velocities deepen the 1st overtone band of CO more than the 2nd overtone band, because line saturation is more important in the former than in the latter (e.g. Origlia et al. 1993, 1997, and refs. therein).

On the left panel of Fig. 5, the changes of the 1st overtone $\mathrm{CO}$ band at $2.29 \mu \mathrm{m}$ with gravity, temperature and surface abundances are shown. As commonly found, CO increases with 

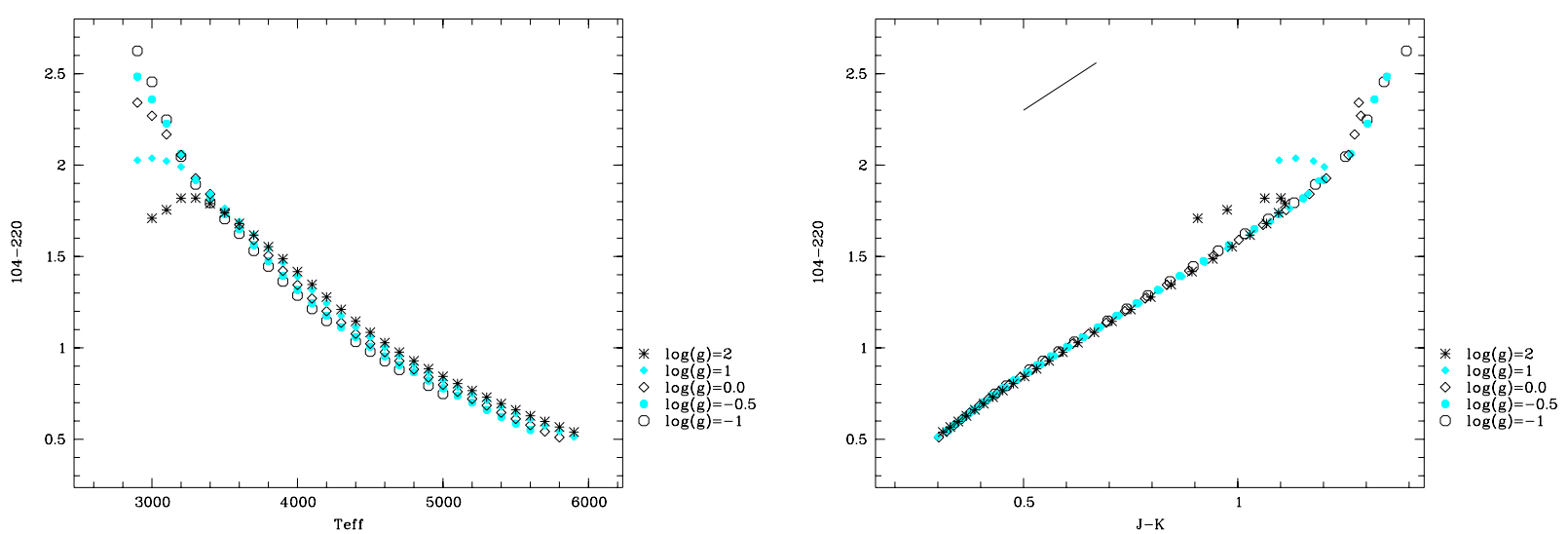

Fig. 3. Temperature sensitive near-IR colours (solar abundances, $15 M_{\odot}$ ). In the right panel, the effect of extinction on cool stellar spectra is shown for $A_{\mathrm{V}}=1$ (using the extinction law of Cardelli et al. 1989, with $R_{\mathrm{V}}=3.1$ ).
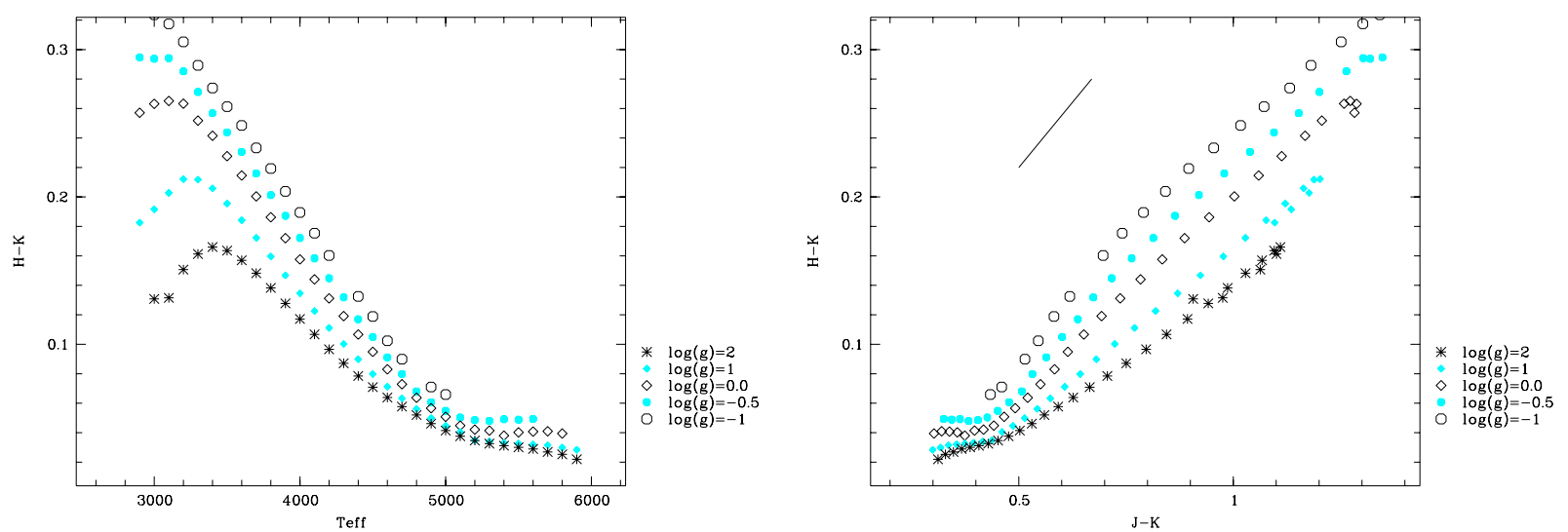

Fig. 4. Gravity sensitivity of $H-K$ (solar abundances, $15 M_{\odot}$ ). Extinction vector as in Fig. 3.
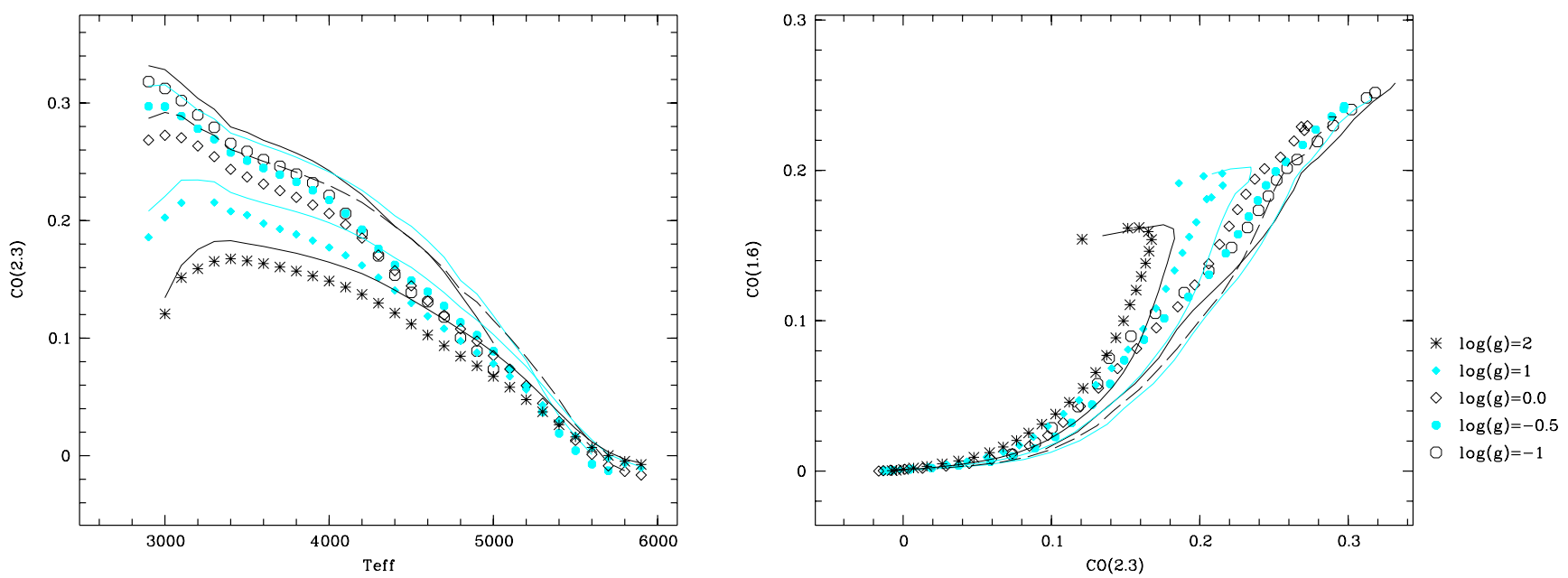

Fig. 5. Measurements of the strength of the 1st overtone CO band at $2.29 \mu \mathrm{m}$ and of the 2nd overtone CO band at $1.62 \mu \mathrm{m}$ in the model spectra $\left(15 M_{\odot}\right)$. Symbols: temperature sequences at the indicated gravities, for RSG-specific abundances. Lines: corresponding sequences for solar-scaled abundances (black and light-coloured lines alternate, and the dashed line has $\log (g)=0)$. Note that at a given $\mathrm{CO}(2.3)$, xCO (1.6) tends to be weaker in low gravity stars.

decreasing temperatures and gravities. The $\mathrm{CO}$ strength progressively levels off when $\log (g)$ takes negative values (i.e. the further dependence on $g$ is negligible). Contamination by $\mathrm{H}_{2} \mathrm{O}$ at high $\log (g)$ produces a drop of the CO index below $3200 \mathrm{~K}$. Switching from solar to RSG-specific abundances reduces the $\mathrm{CO}$ strength generally by small amounts. The effect is maximum around $4500 \mathrm{~K}$ in low gravity models: RSG-specific models with $\log (g)=-1$ at $4500 \mathrm{~K}$ have the same CO index as solar abundance models with $\log (g)=2$, or alternatively with $\log (g)=-1$ but $4800 \mathrm{~K}$.

The effects of $\log (g), T_{\text {eff }}$ and abundances on the apparent strength of the $2 \mathrm{nd}$ overtone $\mathrm{CO}$ band at $1.62 \mu \mathrm{m}$ are similar, 

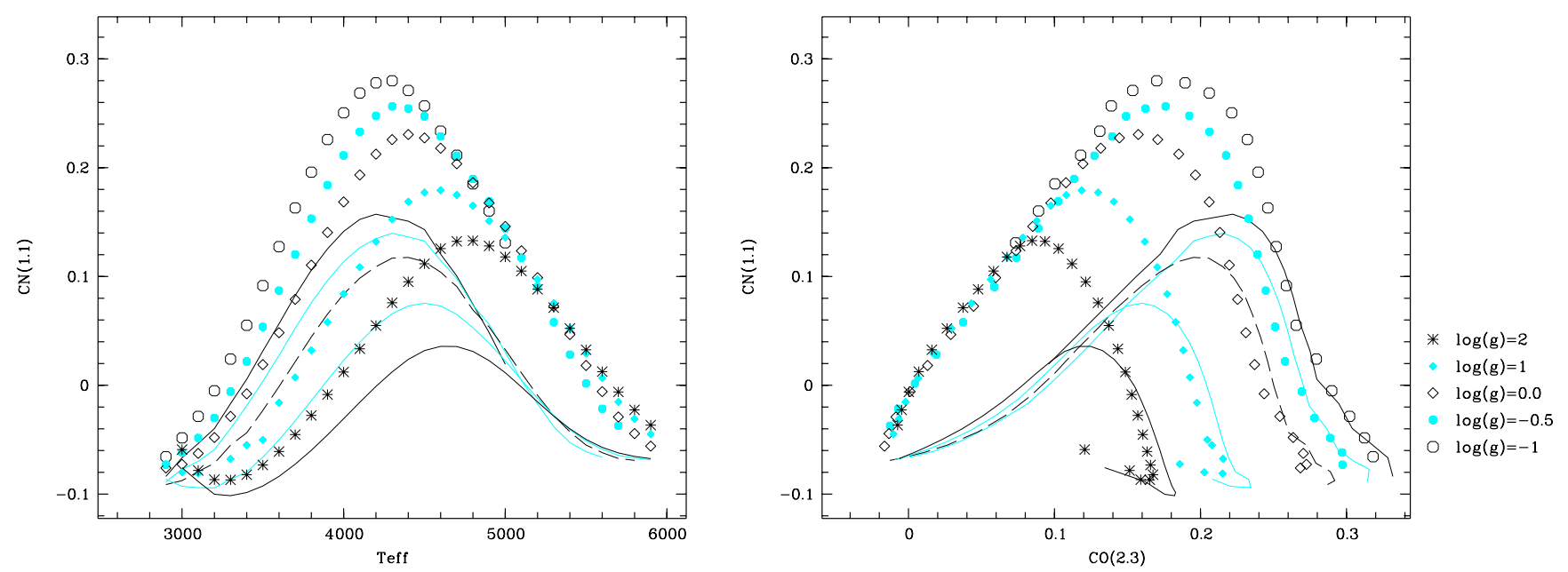

Fig. 6. Measurements of the strength of the $\mathrm{CN}$ band at $1.1 \mu \mathrm{m}$ in the model spectra $\left(15 M_{\odot}\right)$. Symbols and lines are as in Fig. 5 . The main effect of a decrease of mass $\left(15 \rightarrow 1 M_{\odot}\right)$ is a shift of the models at $\log (g) \leq 0$ to the left by up to 0.04 mag in the right hand panel.

with two notable exceptions. First, the effects of changes in the abundance ratios are smaller than at $2.29 \mu \mathrm{m}$. Second, low gravity saturation is reached earlier. The result is summarized in the right panel of Fig. 5. In particular, low gravity stars tend to have weaker $\mathrm{CO}$ bands around $1.6 \mu \mathrm{m}$ than high gravity ones, at a given strength of the $2.29 \mu \mathrm{m}$ band. Contamination of the $H$-band fluxes by $\mathrm{CN}$ absorption contributes to producing this trend, as already hinted at by Wing \& Spinrad (1970).

Moving down from $15 M_{\odot}$ to $1 M_{\odot}$ has a negligible effect on the near-IR CO bands for $\log (g)>0$. At lower gravities, the $1 M_{\odot} \mathrm{CO}$ bands are weaker than the $15 M_{\odot}$ bands (the effect is stronger for the $2.3 \mu \mathrm{m}$ band than for the $1.6 \mu \mathrm{m}$ bands).

\subsection{2. $\mathrm{CN}$}

$\mathrm{CN}$ displays prominent near-IR absorption bands that have been studied extensively in the context of carbon star models (e.g. Loidl et al. 2001). The $\mathrm{CN}$ bands are prominent in red supergiants as well (White \& Wing 1978). While in carbon stars essentially all surface oxygen is locked into $\mathrm{CO}, \mathrm{CN}$ coexists with other oxides in the atmospheres of red supergiants.

The behaviour of $\mathrm{CN}$ bands with varying model parameters is complex, as shown in Fig. 6. Bessell et al. (1989) describe the decrease of the CN $1.1 \mu \mathrm{m}$ band strength with decreasing effective temperature below $3800 \mathrm{~K}$, as well as its gravity dependence (stronger $\mathrm{CN}$ for lower gravities). Our more extended temperature range shows that the maximum $\mathrm{CN}$ strength is reached between 4200 and $4800 \mathrm{~K}$. Both the location of the maximum and its actual strength depend on surface gravity, and on the chemical composition of the atmosphere. $C N$ bands are strongly enhanced in models with RSG-specific abundance ratios. The effect of mass is small. $\mathrm{CN}$ is also enhanced when larger microturbulent velocities are assumed (Tsuji 1976). In empirical samples, spectra with strong $\mathrm{CN}$ absorption bands compared to their $\mathrm{CO}$ bands are candidates for modified surface abundances.

\subsubsection{Other molecular bands longwards of $1 \mu \mathrm{m}$}

$\mathrm{H}_{2} \mathrm{O}$. $\mathrm{H}_{2} \mathrm{O}$ appears very abruptly in the models below a gravitydependent threshold temperature: $3600 \mathrm{~K}$ at $\log (g)=1$,
$3100 \mathrm{~K}$ at $\log (g)=-1$ (based on measurements in the $K$-band wings of the $\mathrm{H}_{2} \mathrm{O}$ band centered near $1.9 \mu \mathrm{m}$ ). Below this threshold $T_{\text {eff }}$, higher gravities lead to stronger spectral bands. Varying the mass between 1 and $15 M_{\odot}$, or switching to RSG-specific abundances, has only small effects on the $\mathrm{H}_{2} \mathrm{O}$ bands.

TiO. Near-IR TiO bands around $1 \mu \mathrm{m}$ ( TiO $\delta, \Delta v=-1)$ and $1.25 \mu \mathrm{m}$ ( $\mathrm{TiO} \phi, \Delta v=-1)$ appear progressively in the models below a gravity-dependent temperature: $\sim 3600 \mathrm{~K}$ at $\log (g)=1, \sim 3400 \mathrm{~K}$ at $\log (g)=-2$ (based on visual inspection of the spectra in that region). Other nearIR TiO bands longwards of $1 \mu \mathrm{m}$, such as the $\phi, \Delta v=0$ band near $1.12 \mu \mathrm{m}$ are hidden in $\mathrm{CN}$ absorption. Again, varying the mass between 1 and $15 M_{\odot}$, or switching to RSG-specific abundances, has only small effects. We note that the next version of PHOENIX calculations will include an update of the TiO partition function and of the electron $f$-values of the TiO bands for the AMES TiO line list (Schwenke 1998), which appears to improve spectral synthesis results for $\mathrm{M}$ dwarfs (Allard et al., in preparation).

VO. The $1.05 \mu \mathrm{m}$ VO band (VO A-X $\Delta v=0$ ) is significant in the model spectra only at $T_{\text {eff }} \leq 3200 \mathrm{~K}$ for $\log (g) \leq 1$. The effect of mass or abundances is small.

\section{Models versus data in two-colour plots}

In this and the following sections, we compare the models with the data collected by Lançon \& Wood (2000) and Lançon et al. (2007). Both sets provide spectra at a spectral resolving power of order 1000 between $0.97 \mu \mathrm{m}$ (sometimes $0.81 \mu \mathrm{m}$ ) and $2.4 \mu \mathrm{m}$. The first set adds low resolution extensions through the optical range down to $5100 \AA$ for a few of the stars. The merged sample contains luminous stars of spectral types G3I to M5I, G3II to M3II, and G5III to M5III, as well as asymptotic giant branch variables for comparison.

As shown in Fig. 7, the agreement between models and data is excellent in near-IR two-colour plots once extinction has been accounted for (see Sect.5). Note that in this and the following figures the lines join models at constant $\log (g)$, while the data should follow real red giant or red supergiant 

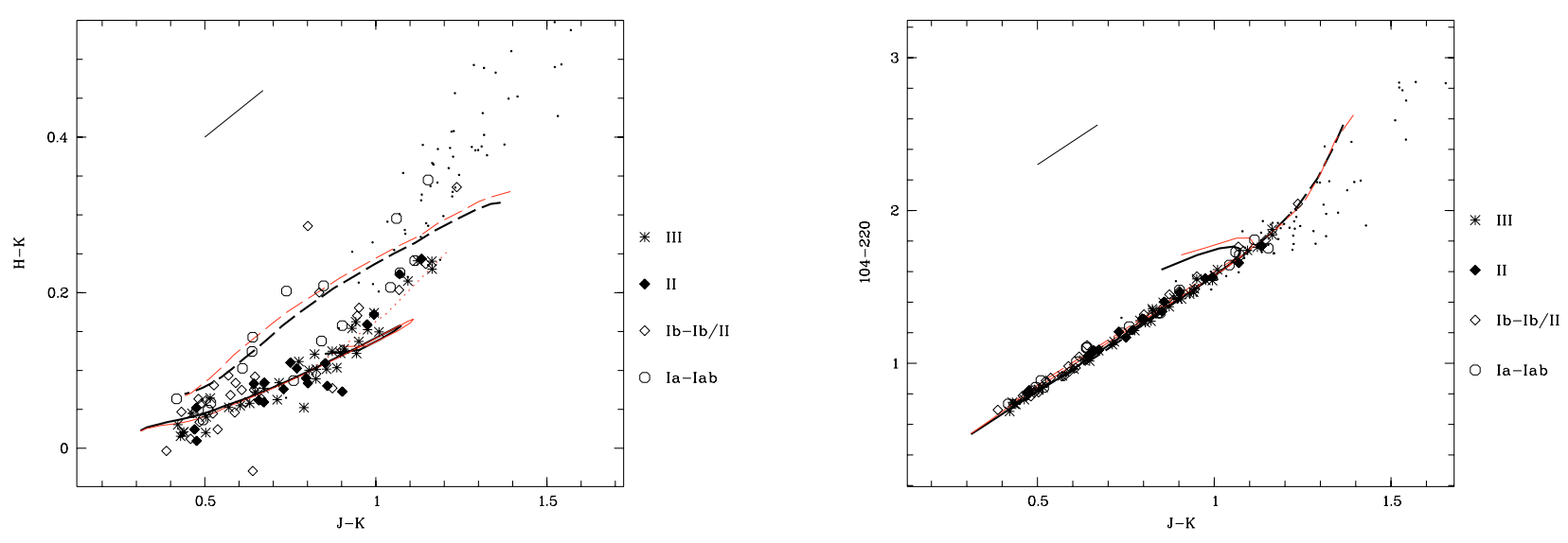

Fig. 7. Two-colour plots with observational and calculated data. The thin lines are $T_{\text {eff }}$ sequences for solar abundances, the thick lines are for RSG-specific abundances. Solid lines are at $\log (g)=2$, dashed lines at $\log (g)=-1$. The dotted line follows models along an illustrative red giant branch at solar metallicity $\left(\left[T_{\mathrm{eff}}(\mathrm{K}), \log (g)\right]=[4200,2],[3800,1],[3400,0]\right)$. Symbols as explained are measurements on dereddened versions of the spectra of Lançon et al. (2007) (see Sect. 5), and dots O-rich Miras from Lançon \& Wood (2000) (not dereddened). The reddening vectors are as in Fig. 3.
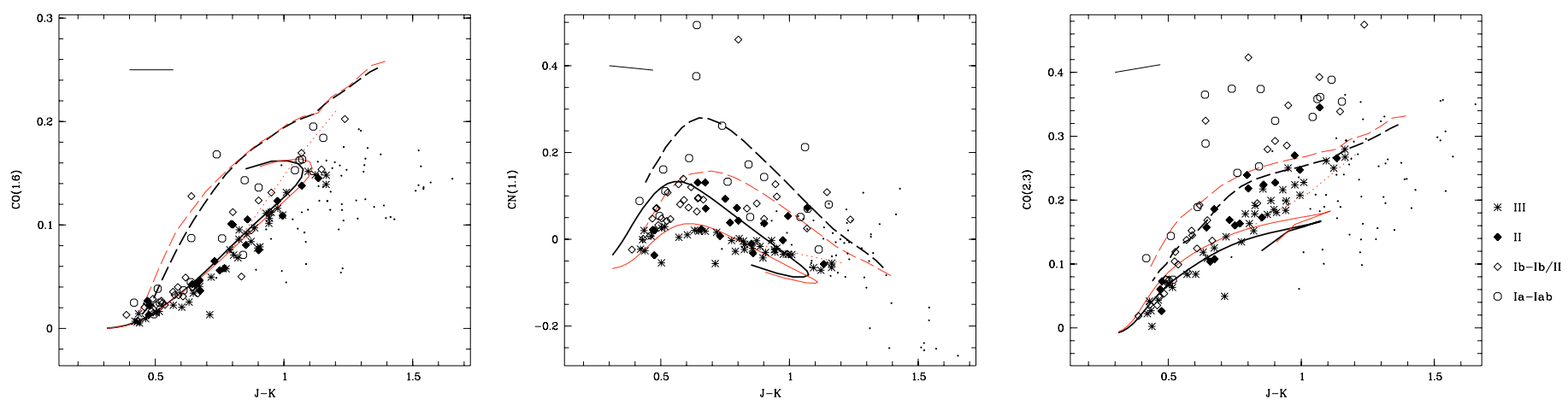

Fig. 8. Plots of molecular indices vs. colours with dereddened data. Symbols and lines are as in Fig. 7.

branches. Evolutionary tracks predict that the warmer giants have $\log (g) \geq 2$ while the cool ones reach $\log (g) \simeq 0$. Red supergiants of various luminosity classes are expected to have $\log (g) \leq 0.5$.

Figure 8 combines measurements of the first and second overtone $\mathrm{CO}$ bands and the $1.1 \mu \mathrm{m} \mathrm{CN}$ band with $J-K$. Agreement between solar metallicity models and empirical data is good for giant stars. The strongest offset is noted for the second overtone $\mathrm{CO}$ bands in the $\mathrm{H}$ window, which tend to be too strong in the cool giant star models. The figures also suggest that modeled first overtone $\mathrm{CO}$ bands might be slightly too weak at low $T_{\text {eff. }}$. Because extinction affects $J-K$, two-index diagrams with a negligible sensitivity to reddening are presented in Fig. 9. The same conclusions hold. The CO line list data are from Goorvitch \& Chackerian (1994a,b), and are known to work very well in the case of $\mathrm{M}$ dwarfs. Therefore, it is unlikely that the line list data for $\mathrm{CO}$ is the cause of the $\mathrm{CO}$ band discrepancies. We note that ${ }^{13} \mathrm{CO}$ contributes to the measured strength of the first overtone $\mathrm{CO}$ bands, and that changes in the ${ }^{13} \mathrm{C}$ abundances induced by stellar evolution may be responsible for some systematic effects. Slightly larger micro-turbulent velocities could improve the ratio of the first to the second overtone $\mathrm{CO}$ band strengths, but would also affect the $\mathrm{CN}$ bands. The outlier giant star near $J-K=0.7$ with weak band strengths is the only
Population II star of the observed sample (HD 218732). Eye inspection of its spectrum reveals a metal poor atmosphere immediately. By contrast, the other giant stars appear to form a reasonably homogeneous sample in terms of metallicity.

While the trends with gravity present in the predicted molecular bands agree qualitatively with those observed, the molecular bands of only a fraction of the observed red supergiants can be reproduced quantitatively. Models with RSG-specific abundances are favoured for a significant number of these objects, which show stronger $\mathrm{CN}$ bands than the solar metallicity models can produce. However, the $\mathrm{CN}$ and $\mathrm{CO}$ measurements show that despite the improvment achieved with the adopted changes in surface abundances, the model parameters explored here are not able to account for the whole range of molecular band strengths observed. Models with larger micro-turbulent velocities reach further into some of the areas occupied by real red supergiants, justifying the ongoing extension of the model grid. Alternatively, some red supergiants in nature may require even higher ${ }^{14} \mathrm{~N}$ abundances than tested here or effective gravities lower than $\log (g)=-1$.

Comparisons between models and data on the star-by-star basis are provided in the following section. 

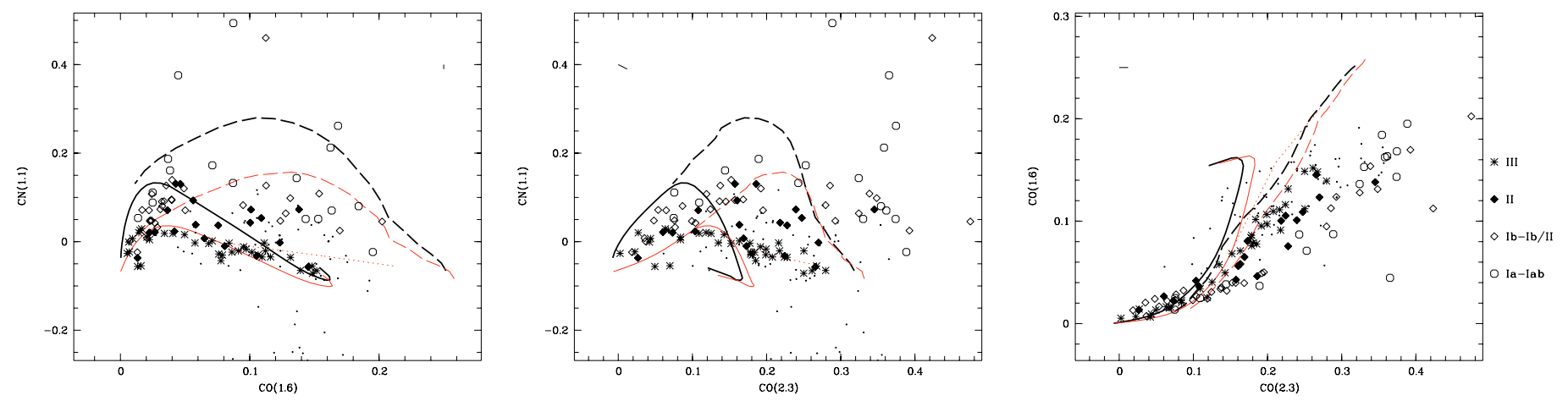

Fig. 9. Molecular index plots with dereddened data. Symbols and lines are as in Fig. 7.

\section{Direct comparison between observed and theoretical spectra}

\subsection{Method}

The comparison between models and data is based on the computation of reduced $\chi^{2}$ differences. The theoretical spectra are smoothed to the resolution of the data, using Gaussian broadening functions with adequate widths (note that for the optical spectra, whose resolution was seeing-dependent, we adopt a single smoothing value which in some cases underestimates the actual $\Delta \lambda$ ). They are then resampled to the wavelength step of the data, i.e. $5 \AA$. A window function is used to eliminate the spectral intervals most strongly affected by telluric absorption, around $1.15,1.4$ and $1.9 \mu \mathrm{m}$.

The rms noise of the data is modelled as being proportional to the square root of the signal. Numerical values given below assume an average signal-to-noise ratio of 50. This simple noise model is a reasonable representation of the typical high frequency noise of the data. The additional uncertainties due to flux calibration errors are not explicitely accounted for. They lead mainly to uncertainties in the estimated extinction values. A further discussion of the effects of the weighting of the data is provided in Sect. 6.1.

A mass of $1 M_{\odot}$ is assumed for giants and bright giants, a mass of $15 M_{\odot}$ for supergiants (see Sect. 6). For each empirical spectrum, the adopted algorithm loops through all model temperatures and gravities (separately for the two sets of abundances). At each of these points, it also loops through an adequate set of extinctions (using the extinction law of Cardelli et al. 1989, with $R_{\mathrm{V}}=3.1$ ), and minimizes the $\chi^{2}$ with respect to the extinction parameter $A_{\mathrm{V}}$. The step in $A_{\mathrm{V}}$ is of $0.05 \mathrm{mag}$. A $\chi^{2}$ map is produced in $T_{\text {eff }}-\log (g)$ space, and the 9 best fits (of the $T_{\mathrm{eff}}-\log (g)-A_{\mathrm{V}}$ space) are plotted for inspection. The $\chi^{2}$ value of the 9 th best fit is typically higher than for the 1 st best fit by $10 \%$ when the best fits are good, and by only a few percent when they are poor. Typical uncertainties on the derived parameters in the case of good fits are $\pm 100 \mathrm{~K}$ in $T_{\text {eff }}$, and \pm 0.2 in $A_{\mathrm{V}}$. For these good fits, gravity is usually determined to better than one step within our set of models $(\log (g)=$ $-1,-0.5,0,1,2)$. Preliminary models with micro-turbulent velocities larger than $2 \mathrm{~km} \mathrm{~s}^{-1}$ were tested only for supergiant star spectra and, in this paper, they are only discussed in cases where the initial fits were poor.

The method is robust with respect to errors in the positions of the individual molecular lines that jointly define the pseudocontinuum of the spectra at the resolution of interest here. Such errors were noted as a difficulty in the measurement of
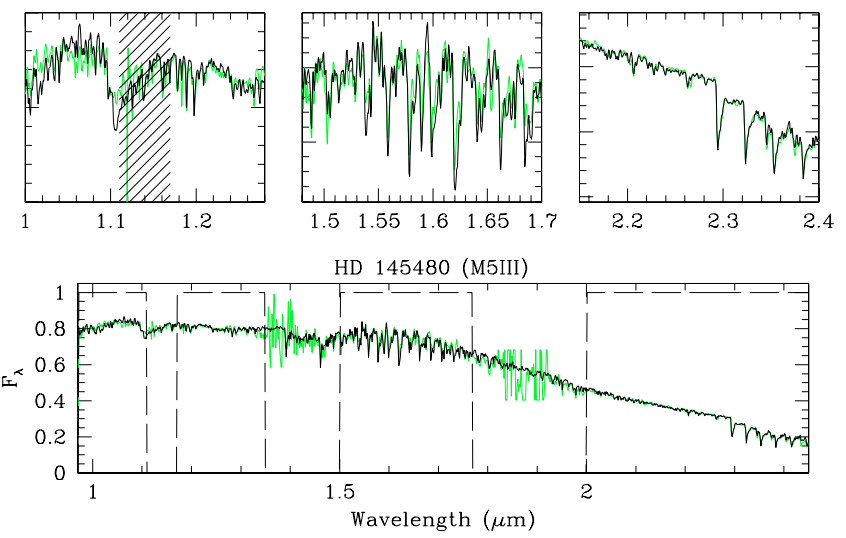

Fig. 10. Fit to a late type giant star spectrum (HD 145480, M5III). The data are shown as dotted lines, the model as a solid line. The window function for the $\chi^{2}$ fit is also shown. Model parameters are $3400 \mathrm{~K}$, $\log (g)=0, A_{\mathrm{V}}=0.4, \chi^{2}=1.7$. Such a fit quality is typical for all the giants without available optical spectra.

individual metal lines by Vanhollebeke et al. (2006). In order to verify this, we added random noise to the data at the level of a few percent, i.e. enough to completely alter the apparent positions of the blended CN lines between 2 and $2.3 \mu \mathrm{m}$. Differences in the derived parameters were well within the uncertainties stated above.

\subsection{Giant stars}

The data sample contains 35 stars of class III with spectral types G5 to M5 (after elimination of one particularly uncertain luminosity class and one known metal poor star with obviously weaker spectral features). Their shortest wavelength is $0.97 \mu \mathrm{m}$, except for 5 spectra with an optical extension.

Good fits are obtained for essentially all the near-IR spectra with the solar metallicity models. An example is given in Fig. 10. Among the satisfactory fits, there is a tendency for $\chi^{2}$ to increase systematically from about 1 for types $\leq \mathrm{K} 4\left(\chi^{2}=\right.$ $0.6-1.4$, depending on the actual $\mathrm{S} / \mathrm{N}$ of individual spectra) to about $2(1.5-2.5)$ for the $M$ stars. This trend is due to a wealth of weak lines and deeper molecular bandheads at low temperatures, which among others induces a higher sensitivity of the value of the $\chi^{2}$ to residual wavelength calibration errors or model line lists.

The $\chi^{2}$ values for combined optical+near-IR spectra take values of 3 to 7 for satisfactory fits (considering the sensitivity of the $\chi^{2}$ to the smoothing parameter at optical wavelengths and to 


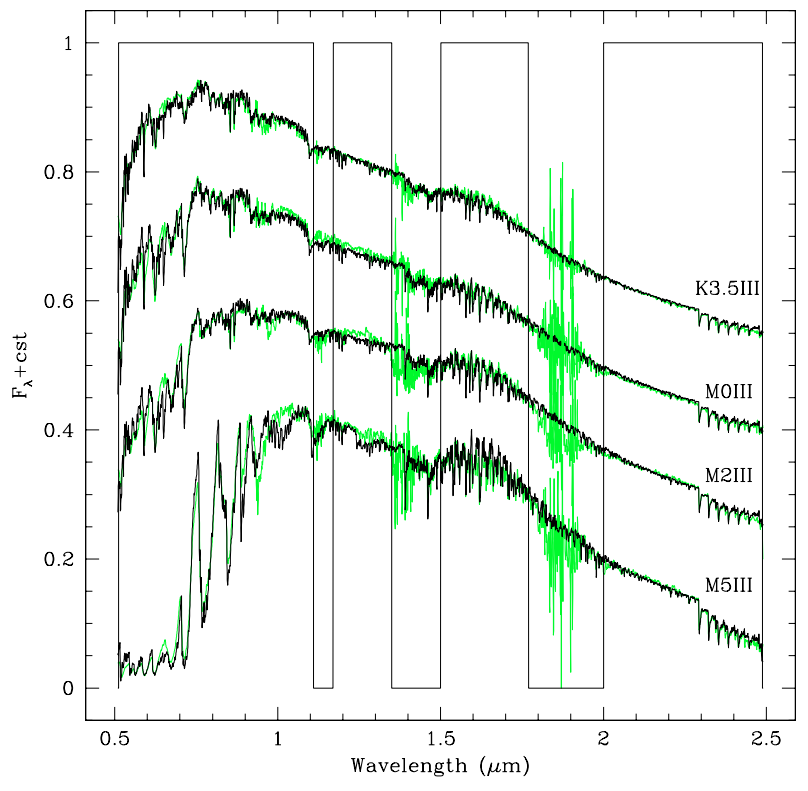

Fig. 11. Best fits to four giant star spectra that extend to optical wavelengths. Data are shown as dotted lines, best fit models as solid lines. The window function used to reject the noisiest spectral regions is also shown. From top to bottom: K3.5III star BS 4432 with model [ $T_{\text {eff }}$, $\left.\log (g), A_{\mathrm{V}}, \chi^{2}\right]=[4100 \mathrm{~K}, 2,0.55 \mathrm{mag}, 2.3]$; M0III star BS 4371 with [3900 K, 1, $0.2 \mathrm{mag}, 4.5]$; M2III star BS 5301 with [3800 K, 1, $0.55 \mathrm{mag}, 5.2$ ]; M5III star BS 4267 with [3300 K, $1,-0.3 \mathrm{mag}, 13.9]$.

flux calibration errors over such an extended wavelength range). Examples are shown in Fig. 11. The best fit to the spectrum of the coolest giant, the M5III star BS 4267 (=HD 94705) requires marginally negative extinction. Considering the flux calibration uncertainties and the choice of one particular extinction law, such a result is not alarming. Cooler models by only $100 \mathrm{~K}$ or models with higher $\log (g)$ by 0.5 would result in a positive value of the estimated $A_{\mathrm{V}}$. The most obvious shortcoming of the models for giants this cool is an overprediction of the $\mathrm{TiO}$ absorption bands near 1 and $1.25 \mu \mathrm{m}$. follows:

The results of the fitting procedure can be summarized as

Temperatures range from $5300 \mathrm{~K}$ for type G5, to $3300 \mathrm{~K}$ for type M5. As expected from stellar evolution tracks, the highest available gravity $(\log (g)=2)$ is selected for giants earlier than K7 (with one exception), then progressively more spectra are assigned $\log (g)=1$ and later $0 . A_{\mathrm{V}}$ values are spread between 0 and 1 (with 4 cases of marginally negative values). No correlation is found between $A_{\mathrm{V}}$ and $T_{\mathrm{eff}}$.

Adopting the models with RSG-specific abundances rather than solar ones leads to poorer fits in all but one case. The values of the reduced $\chi^{2}$ increase by 0.5 units on average. While the distribution of estimated effective temperatures for the sample is relatively flat with the assumption of solar abundances, it becomes bimodal with RSG-specific abundances: temperatures between 4500 and $5000 \mathrm{~K}$ are systematically avoided, because the $\mathrm{CN}$ bands of these models are too strong at the surface gravities of giant stars (cf. Fig. 6). This result was expected from Figs. 9 and 8. It is satisfactory as our set of RSG-specific abundances is not designed to match abundances in giant stars.

\subsection{Bright giants}

The sample contains 29 bright giants of class Ib/II or II. Spectral types range from G3 to M3. None of the corresponding

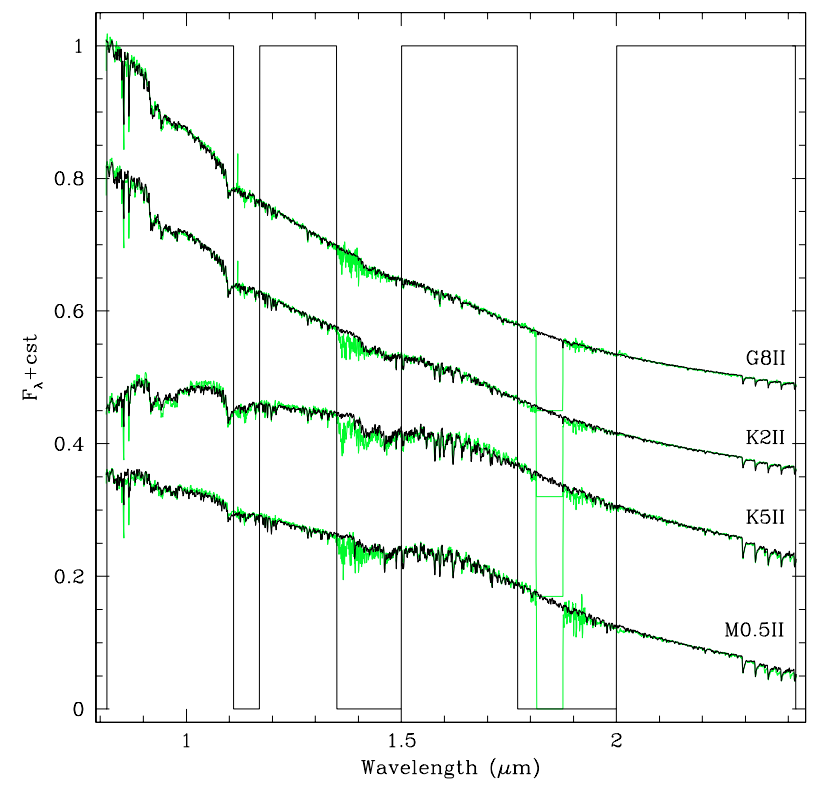

Fig. 12. Best fits to four bright giant star spectra (class II) that extend down to $0.81 \mu \mathrm{m}$. Figure set-up is as in Fig. 11. From top to bottom: G8II star HD 150416 with $\left[T_{\mathrm{eff}}, \log (g), A_{\mathrm{V}}, \chi^{2}\right]=[5000 \mathrm{~K}, 1,0.1 \mathrm{mag}$, 0.65]; K2II star BD-292374 with [4500 K, 2, -0.15 mag, 0.65]; K5II star HD 168815 with [4100 K, 1, 1.3 mag, 1.8]; M0.5IIb star HD 132933 with $[4000 \mathrm{~K}, 2,0.4 \mathrm{mag}, 1.4]$. Among the above, we classify the K5II fit as satisfactory, the others as very good.

spectra extend through optical wavelengths, but 11 extend down to $8100 \AA$. Their properties in terms of colours and molecular indices are spread between those of giants and supergiants. On average, they display slightly stronger bands of $\mathrm{CO}$ and significantly stronger bands of $\mathrm{CN}$ than giants of class III, at a given (dereddened) colour.

The solar metallicity model fits to all the spectra are satisfactory, two thirds of them being very good (Fig. 12). The models clearly contain all the molecular bands required. Marginally negative values of $A_{\mathrm{V}}$ are obtained in four cases, which is again not unexpected considering flux calibration uncertainties. The most common shortcomings found when the fits are not perfect are the following:

- There is a tendency for the models to show stronger $\mathrm{CO}$ bands at $2.29 \mu \mathrm{m}$ and weaker $\mathrm{CN}$ bands at 0.93 and $1.1 \mu \mathrm{m}$ than observed. This problem is only detectable clearly when the data extend down to $0.81 \mu \mathrm{m}$.

- For stars with spectral types around K5II whose spectra extend down to $0.81 \mu \mathrm{m}$, the models struggle to reproduce the energy distribution around $1 \mu \mathrm{m}$, where it peaks between deep $\mathrm{CN}$ bands. This difficulty is certainly related to the strength of the $\mathrm{CN}$ bands at those temperatures (see Fig. 6).

- In two cases (spectral type M0Ib/II and M0.5II), the model $\mathrm{CN}$ bands are too weak while $\mathrm{CO}$ is reproduced well (Fig. 13).

The $T_{\text {eff }}$ and $\log (g)$ scales obtained for the bright giants have a scatter similar to those found for giants and supergiants and are located between the two, as expected. Bright giants with spectral types earlier than K4 (included) are assigned $\log (g)=2$ (with one exception: HD 170457, G8Ib/II), and values of $\log (g)$ for types K5-M3 are scattered between 1 and -0.5 . No correlation is found between $A_{\mathrm{V}}$ and $T_{\text {eff }}$.

When moving from solar abundances to RSG-specific abundances, the $\chi^{2}$ test indicates that the fits are degraded in a 


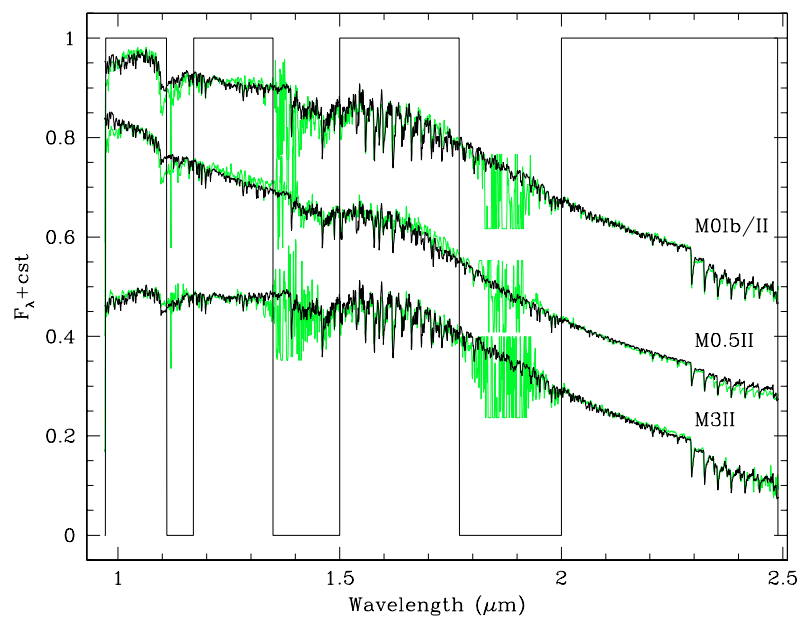

Fig. 13. Best fits to two bright giant star spectra for which the $\mathrm{CN}$ band at $1.1 \mu \mathrm{m}$ is particularly poorly reproduced, and of the coolest class II star of the sample. Figure set-up is as in Fig. 11. From top to bottom: M0Ib/II star HD 145384 with $\left[T_{\text {eff }}, \log (g), A_{\mathrm{V}}, \chi^{2}\right]=[3400 \mathrm{~K},-0.5$, $-0.15 \mathrm{mag}, 3.2]$; M0.5II star HD 142676 with [3900 K, 2, $0.0 \mathrm{mag}, 4.0$ ]; M3II star HD 153961 with [3500 K, 0, $1.0 \mathrm{mag}, 3.3$ ].

majority of cases (typically by $0.5 \chi^{2}$ units, as for the sample of class III stars). However, a significantly improved $\chi^{2}$ is obtained for 4 stars, and the $\chi^{2}$ changes are insignificant in 7 cases.

The improvements correspond to four stars of type K2 to M0 (out of the 11 bright giants available in this range), with estimated $T_{\text {eff }}$ of 4300 to $3400 \mathrm{~K}$. Eye inspection of the corresponding four spectra shows that the decrease in $\chi^{2}$ corresponds to a better fit to the $\mathrm{CN}$ bands, which were not deep enough (by small amounts) in the solar metallicity models. In some cases, the improved $\chi^{2}$ was associated with a decrease in $T_{\text {eff }}$ by $100 \mathrm{~K}$ or an increase of $\log (g)$ by one bin size, but statistics are too small to define significant trends.

Degraded fits are frequently associated with excessive strengths of the model CN bands when the RSG-specific abundances are used. The $T_{\text {eff-distribution obtained with the }}$ RSG-specific abundances still shows a zone of avoidance between 4500 and $5000 \mathrm{~K}$, but the effect is not as obvious as in the case of class III stars. Although small number statistics affect this result, we note that all class II spectra with estimated $T_{\text {eff }}$ in that range have poorer fits with the RSG-specific abundances than with solar ones. As expected, models with the adopted RSG-specific abundances do not apply to the majority of class II stars but they do allow us to identify candidate objects that may have suffered more than standard dredge-up.

\subsection{Supergiant stars}

The data sample contains 37 spectra of stars of class I, Ia, Iab or Ib (after removal of one particularly odd case that is probably misclassified and of one spectrum with poor correction for telluric absorption). Spectral types range from G2 to M5. The stars with the latest spectral types $(\geq \mathrm{M} 2)$ are all known or suspected variables (as are the vast majority of late type supergiants in nature). 9 spectra, all with spectral type M, extend through the optical range; note that the optical and near-IR spectra of individual objects were taken within less than three weeks of each other. 8 spectra extend down to $0.81 \mu \mathrm{m}$.

Good fits to the red supergiant spectra with solar metallicity models are obtained for 13 of the 37 spectra, all of which are of spectral type G2-G8. 16 of the remaining spectra find a model

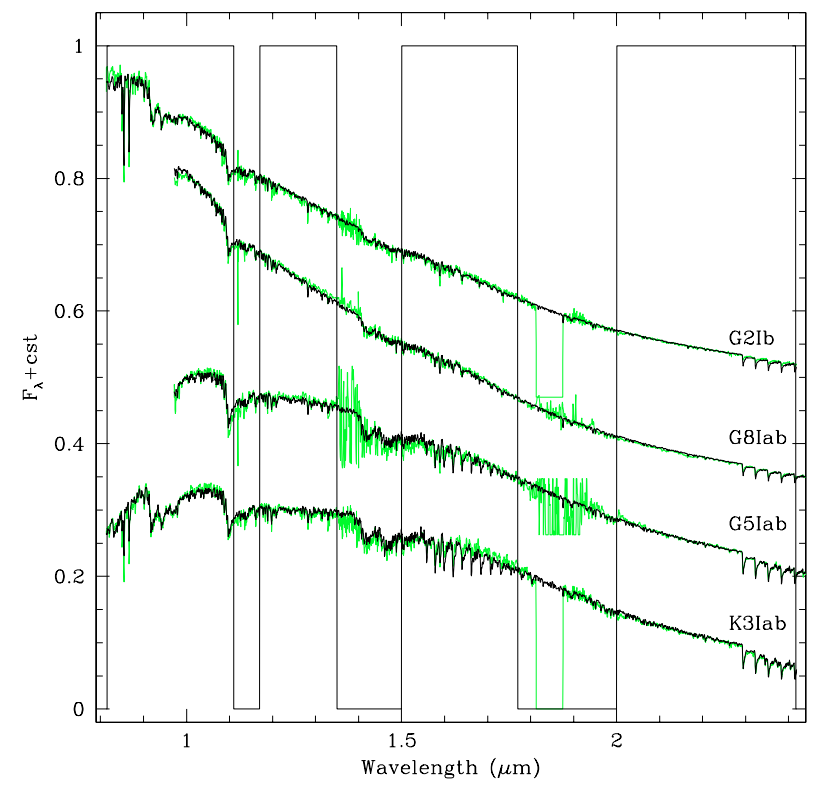

Fig. 14. Good and reasonable best-fits to warm red supergiant spectra (class I). Figure set-up is as in Fig. 11. Abundances are solar. From top to bottom: G2Ib star HD 182296 with $\left[T_{\mathrm{eff}}, \log (g), A_{\mathrm{V}}, \chi^{2}\right]=[5000 \mathrm{~K}$, 0, $0.95 \mathrm{mag}, 1.3$ ]; G8Iab star HD 206834 with [4900 K, 1, $0.3 \mathrm{mag}$, 0.85]; G5Iab star HD 170234 with [4500 K, 0, $2.15 \mathrm{mag}, 1.8$ ]; K3Iab star HD 187238 with [4100 K, 0, $1.65 \mathrm{mag}, 2.2]$. We have not counted the K3Iab case as a good fit, because the model CO bands around $1.7 \mu \mathrm{m}$ are too strong. Note that the spectral type of the second and third stars are likely to be incorrect.

representation that is still reasonable (though often significantly poorer than the fits we called satisfactory within class II above). These are spread over the whole range of spectral types and include some of the data that extend through optical wavelengths. In general, stars of luminosity class Ib are easier to fit than those of class I, Ia or Iab, and all class Ib stars of our sample can be matched well or reasonably well. Finally, we classify 7 of the red supergiant fits as poor. Five of these correspond to variable stars with spectral types later than M3.5 (class I, Ia or Iab), the two others are of spectral type M0Ia and G5Ia.

Figures 14-16 illustrate some of the good, intermediate and poor model fits.

The main shortcomings of the models when the fits are of intermediate quality are the following:

- A relatively common feature is a shortage of flux in the models around $1 \mu \mathrm{m}$, as seen in two spectra of Fig. 15. This problem was already mentioned for a few bright giants of class II, as a property that is associated with strong $\mathrm{CN}$ bands and can be identified only when the observed spectra extend far enough to short wavelengths.

- Even when the 1 st overtone CO bands after $2.29 \mu \mathrm{m}$ are reproduced reasonably well, it happens that the relative strengths of the 2 nd overtone $\mathrm{CO}$ bands in the $\mathrm{H}$ window are incorrect, the transitions at longer $\mathrm{H}$ window wavelengths $(1.65-1.75 \mu \mathrm{m})$ being too strong in the models compared to the data (last spectrum of Fig. 14 and 2nd and 3rd spectrum of Fig. 15).

- In the coolest models, bands of $\mathrm{TiO}$ appear (in particular near $1.25 \mu \mathrm{m}$ ) that are not seen in the data.

Poor fits are obtained for the coolest stars (e.g. bottom spectrum of Fig. 16) and for stars with extreme CN bands (e.g. top three spectra of Fig. 16). We recall that the coolest stars are also 


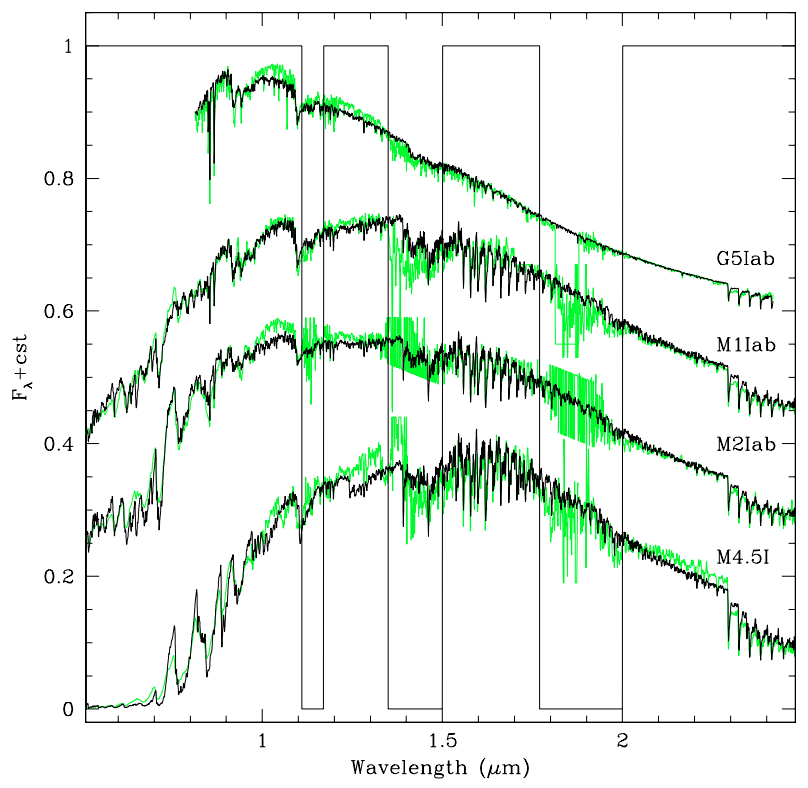

Fig. 15. A selection of marginally acceptable best-fits to red supergiant spectra. Figure set-up is as in Fig. 14. From top to bottom: G5Iab star HD 165782 with $\left[T_{\mathrm{eff}}, \log (g), A_{\mathrm{V}}, \chi^{2}\right]=[4900 \mathrm{~K},-1,2.4 \mathrm{mag}$, 2.8]; M1 Iab star HD 98817 with [3700 K, $-1,1.8 \mathrm{mag}, 7.8$ ]; M2Iab star BS 3364 (= HD 72268) with [3500 K, -0.5, $1.0 \mathrm{mag}$, 12.4]; M4.5I star V774 Sgr with [3200 K, $-0.5,1.7 \mathrm{mag}, 22.7]$.

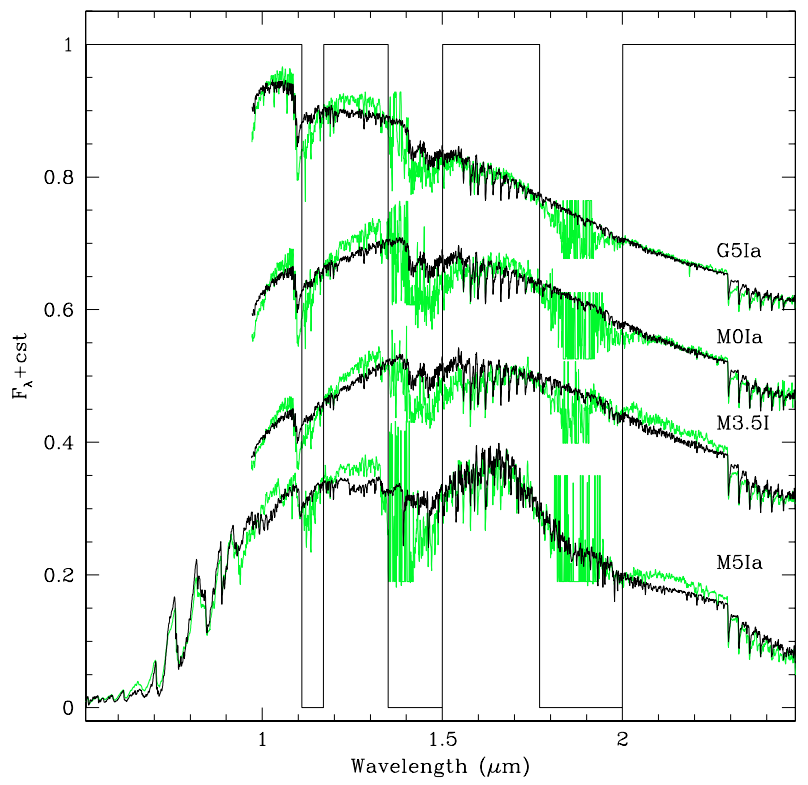

Fig. 16. A selection of poor best-fits to red supergiant spectra. Figure set-up is as in Fig. 14. From top to bottom: G5Ia star HD 155603 (classified K0 0-Ia by Keenan \& McNeil 1989) with $\left[T_{\text {eff }}, \log (g), A_{\mathrm{V}}\right.$, $\left.\chi^{2}\right]=[4300 \mathrm{~K},-0.5,1.8 \mathrm{mag}, 8.6]$; M0Ia star Trumpler $1-27$ with [4200 K, -1, $4.4 \mathrm{mag}, 13.4]$; M3.5I star IRC -20427 with [4000 K, -1, 5.35 mag, 15.2]; M4-5Iab star CL Car with [3300 K, 2, 1.65 mag, 21.6]. The fits being of poor quality, the derived parameters are not reliable and are given here only for the sake of completeness.

variable and that discrepancies are to be expected in a comparison with static models. When the $\mathrm{CN}$ bands are strong, the derived temperatures are a compromise between the necessity to reproduce the energy distributions and the CO bands at $2.29 \mu \mathrm{m}$ (which pulls towards low temperatures), and the need to maximize CN depths (which pulls towards $4100 \mathrm{~K}$, cf. Fig. 6). When optical spectra are taken into account, the relative weight of the
$\mathrm{CN}$ bands is reduced compared to $\mathrm{CO}$, optical features and the energy distribution. On the contrary, when only wavelengths between 0.97 and $2.4 \mu \mathrm{m}$ are available the rôle of the $\mathrm{CN}$ bands is large. This explains why in Fig. 16 such a large difference in $T_{\text {eff }}$ is obtained between the M3.5Ia star (no optical spectrum, best fit $T_{\text {eff }}=4000 \mathrm{~K}$ ) and the M4-5I star (optical spectrum available, best fit $T_{\text {eff }}=3300 \mathrm{~K}$ ). The temperatures of the M0Ia and M3.5I stars of that figure are most probably overestimated. For a similar reason, the temperature of the G5Ia star at the top of the figure may be underestimated (compare with the G5Iab star in Fig. 15).

A typical problem with the best fit models for the spectra with very strong $\mathrm{CN}$ is the relative strength of the various CO bands. The G5Ia star HD 155603 (Fig. 16) provides the most extreme example. It has the strongest $2.29 \mu \mathrm{m} \mathrm{CO}$ band of our whole supergiant sample and among the strongest $\mathrm{CN}$ bands as well, but in the $\mathrm{H}$ window $\mathrm{CO}$ is almost absent. None of the current models with $v_{\text {mic }}=2 \mathrm{~km} \mathrm{~s}^{-1}$ reproduces this combination. Models with larger micro-turbulent velocities improve the representation of these extreme spectra.

Water bands are another cause of disagreement between models and data. The near-IR bands of $\mathrm{H}_{2} \mathrm{O}$ and $\mathrm{CN}$ overlap in wavelength to such a degree that confusion can occur (and has occurred in the past, cf. Wing \& Spinrad 1970). The shapes of the $1.1 \mu \mathrm{m}$ bands of $\mathrm{H}_{2} \mathrm{O}$ and $\mathrm{CN}$ are subtly different (cf. Fig. 5 of Lançon \& Wood 2000). The bands observed in red supergiants correspond closely to $\mathrm{CN}$, although contamination with $\mathrm{H}_{2} \mathrm{O}$ is possible. The $\mathrm{H}_{2} \mathrm{O}$ band around $1.9 \mu \mathrm{m}$, which is very deep and broad in Miras, is inconspicuous in red supergiants. It may be present at a low level in the coolest ones observed, such as CL Car (Fig. 16), which are semi-regular long period variables. The clearest $\mathrm{H}_{2} \mathrm{O}$ signature in the observed red supergiant spectra is a sharp bandhead at $1.32 \mu \mathrm{m}$, although the detection of this feature requires good corrections for telluric absorption bands. Based on this signature, the 7 coolest supergiants of our sample contain $\mathrm{H}_{2} \mathrm{O}$ (all these are variable). The models however either do not show this bandhead (low $g$ ) or, when they do (high $g$ ), also display a $1.9 \mu \mathrm{m}$ band that is much wider and deeper than observed.

Finally, the semi-regular variables V774 Sgr, EV Car and CL Car (Figs. 15 and 16) have a clear VO absorption band at $1.05 \mu \mathrm{m}$ and small or inexistent absorption bands at $1 \mu \mathrm{m}$ and $1.25 \mu \mathrm{m}$, two properties that are not matched by the models.

When moving from the models with solar abundances to the RSG-specific abundances, the $\chi^{2}$ test indicates that about a third of the fits are improved, another third are degraded, and the quality of the final third of the fits is essentially unchanged.

The deteriorations, when present, are not severe. In most cases, it seems that abundance values intermediate between the adopted solar and RSG-specific sets would provide optimal fits, which is not surprising considering that evolutionary tracks for red supergiants cover a range of abundances. Eye inspection shows that quite a few stars with equally good fits with both model sets also fall in this category.

The improvements obtained with RSG-specific abundances for a fraction of the red supergiants are significant, although they clearly do not resolve all the difficulties. They are associated with a better representation of the observed $\mathrm{CN}$ bands and sometimes also with a better match to the $\mathrm{CO}$ bands around $1.6 \mu \mathrm{m}$ (see also Sect. 5.5). One may distinguish two subcategories of improvements. On one hand, some of the stars that already had reasonable model counterparts with solar abundances have better, often good adjustments with RSG-specific abundances. These are mainly stars of type $\mathrm{G}$ and $\mathrm{K}$. An example is given 


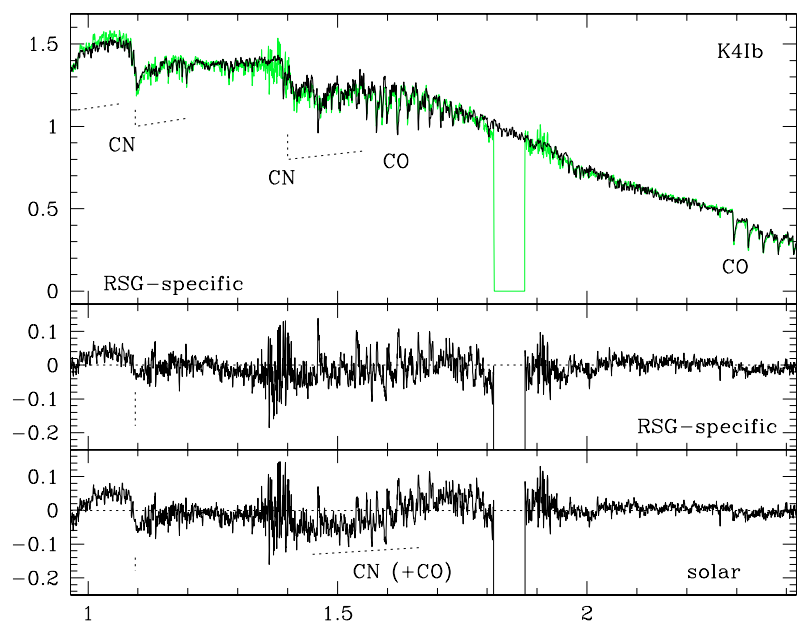

Fig. 17. Top: K4Ib star HD 185622a and best fit model with RSGspecific abundances. Middle: residuals of the fit shown above (datamodel). Bottom: residuals of the best fit with solar metallicity models $\left(\chi^{2} 1.22\right.$ times larger than in the RSG-specific case). Note the $\mathrm{CN}$ residuals below $1 \mu \mathrm{m}$, around $1.1 \mu \mathrm{m}$ and in the slope between 1.45 and $1.75 \mu \mathrm{m}$. These are typical and systematic shortcomings of the solar metallicity models in the cases where RSG-specific models provide a better fit.

in Fig. 17. On the other hand, the improvements refer to stars that had poor fits with solar abundances, and for which the RSG-specific abundances lead to somewhat better but still unacceptable fits. These are the same 7 stars as mentioned earlier. The models cannot simultaneously reproduce their $\mathrm{CO}$ bands (1.6 and $2.29 \mu \mathrm{m}$ ), their $\mathrm{CN}$ bands and their energy distribution. More extended model grids are needed to characterize these objects. Problems related to $\mathrm{H}_{2} \mathrm{O}$, TiO and VO, when present, remain essentially unchanged.

The explored changes in surface abundances induce changes in the best-fit parameters for the sample of observed stars with maximal amplitudes of $\pm 200 \mathrm{~K}$. For the sample as a whole, there is no strong correlation between the change in $T_{\text {eff }}$ and the actual value of $T_{\text {eff }}$, which is not surprising considering that many fits are imperfect and that the behaviour expected from theory is complex (see Sect. 6.2).

The $T_{\text {eff }}$ distribution of the red supergiant sample obtained under the assumption of RSG-specific abundances shows no anomaly. Scrutiny of the 2D distribution of estimated parameters in the $\log (g)-T_{\text {eff }}$ plane suggests that a narrow zone extending diagonally from $\left[\log (g)=0, T_{\text {eff }}=4000 \mathrm{~K}\right]$ to $[\log (g)=1$, $\left.T_{\text {eff }}=5000 \mathrm{~K}\right]$ (with no extension to lower gravities) might nevertheless be underpopulated. The statistical significance of this gap is low because of small sample numbers. Its presence would favour a general picture in which RSG-specific abundances are only relevant to red supergiants with large initial masses or to late stages of red supergiant evolution.

\subsection{Effects of the weighting of various parts of the spectra}

Because there are systematic differences between the best fit models and the observed spectra, the best fit model parameters depend on the weights given to the various spectral features in the fitting procedure. Our standard method weights the data based on a reasonable simplified model for the high frequency noise in the data. This adopted weight is inversely proportional to the square root of the signal, i.e. spectral regions with large fluxes contribute more to the $\chi^{2}$ than regions with small fluxes.
Since the spectra of cool stars peak around $1 \mu \mathrm{m}$ (in the flux density units adopted in this paper), molecular bands near this wavelength are important in the fit. In practice, this weighting makes $\mathrm{CN}$ bands relatively important and CO bands (around $1.6 \mu \mathrm{m}$ and $2.3 \mu \mathrm{m}$ ) comparatively unimportant.

If the noise was indeed Gaussian, uncorrelated between pixels, and exactly of the amplitude assumed, then our procedure would select the models with the largest likelihoods. This is not the case (flux calibration errors, wavelength-dependent gains and contributions of the read-out noise, etc.), and therefore our weighting is in some ways non-optimal. We may choose various alternative methods (see Decin et al. 2004, for an interesting discussion of comparable issues). First, we may decide to fit measurements of the depths of one or several features rather than spectral segments. Unfortunately, the selection of one or the other feature remains somewhat arbitrary. Second, we may decide to focus on either the optical or the near-IR spectral range. This circumvents the difficulty of reproducing the global energy distribution (possible uncertainties in the relative flux calibration of the optical and near-IR data, uncertainties in the adopted extinction law, etc.). Third, we may keep the whole spectrum but explore the effects of alternative weightings. We briefly summarize below the main trends found while investigating these three options.

Figures 18 and 19 show how three important near-IR molecular features in the models compare with their observed counterparts, when our standard weighting procedure is applied to select the best fit. Note that the corresponding figures for $H-K, J-H$, $J-K$ or $104-220$ (not shown) are very well behaved, which only states that the adopted extinction law is capable of dealing with the actual extinction (and with flux calibration errors) rather well.

As expected, systematic drifts away from the perfect match are smaller for the $1.6 \mu \mathrm{m} \mathrm{CO}$ features than for the $2.3 \mu \mathrm{m}$ $\mathrm{CO}$ band, the latter being located in a region of low flux (small contribution to the $\chi^{2}$ ). The best-fit models have systematically deeper $2.3 \mu \mathrm{m}$ CO bands than the data for warm stars (types $\mathrm{G}$ and $\mathrm{K}$ ), but systematically too shallow $2.3 \mu \mathrm{m} \mathrm{CO}$ bands for the coolest stars (type M). By changing the weights in the fitting procedure (e.g. by assuming a constant signal-to-noise ratio), the $2.3 \mu \mathrm{m}$ bands can be reproduced better, but at the cost of a loss of the fit quality at shorter near-IR wavelengths.

The CN bands are reproduced well for giant stars. But they are too shallow in the best fit models for some of the bright giants and for the supergiants. Here, changing the fitting weights has a small effect compared to more fundamental model parameters such as abundances, gravities or micro-turbulence. RSG-specific abundances move the bulk of the red supergiants into a satisfactory location (Fig. 19). With RSG-specific abundances, the fits to $\mathrm{CO}$ bands around $1.6 \mu \mathrm{m}$ are not fundamentally improved or degraded on average, while the first overtone $\mathrm{CO}$ bands $(2.3 \mu \mathrm{m})$ of the best fits become shallower, i.e. too shallow. By assigning $\mathrm{CO}$ more weight in the fits, it is possible to reduce this discrepancy while still observing the global improvement for CN. But with the current grid of models, no fully satisfactory solution can be found for any weighting scheme.

The weights given to various spectral ranges impact on the estimated stellar parameters. Examples have already been given in Sect. 5.4, and further discussions can be found below.

\section{Discussion}

Providing estimates of fundamental stellar parameters is a major application of theoretical spectra. Our discussion focuses on 

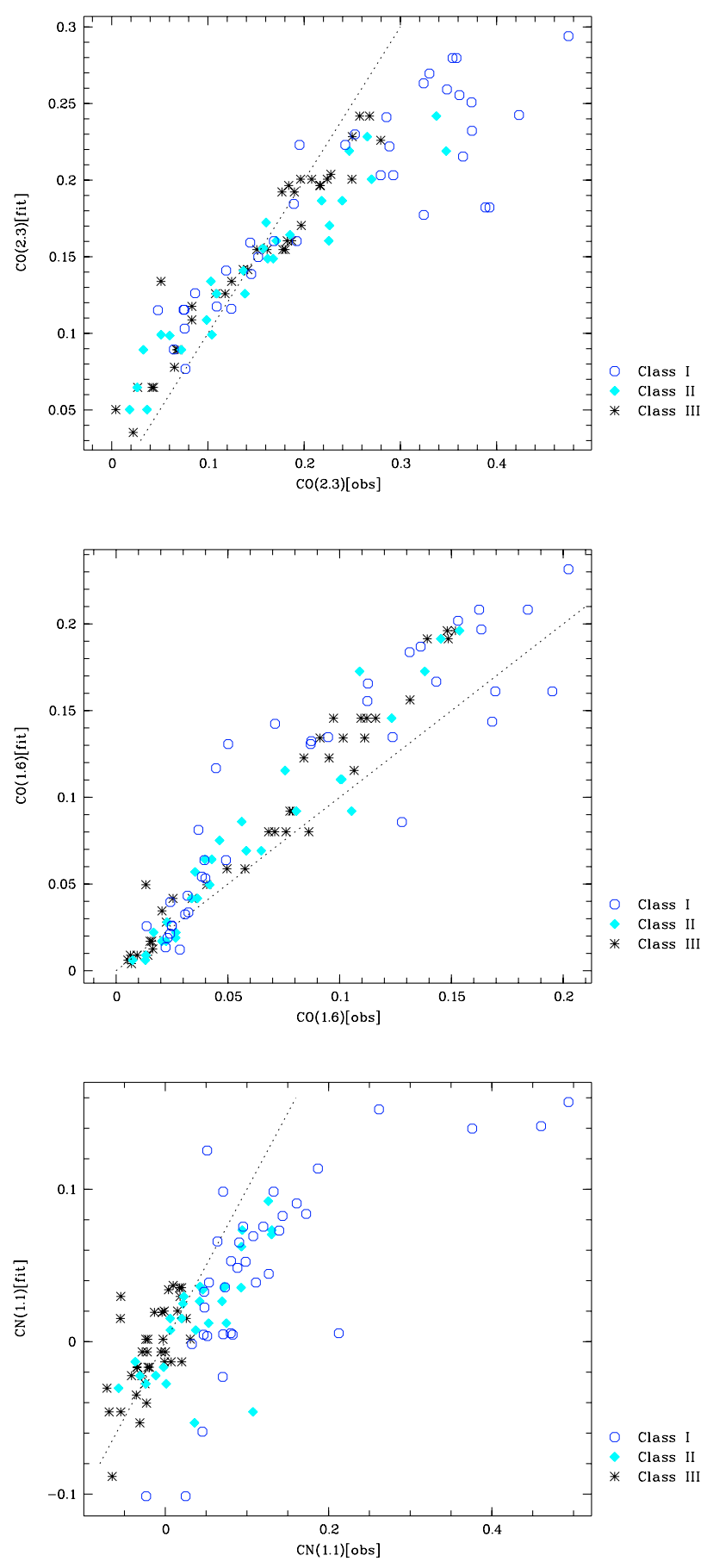

Fig. 18. Top: strength of the $2.3 \mu \mathrm{m} \mathrm{CO}$ band of the best fitting solar metallicity models versus strength of this band in the dereddened observed spectra (cf. Table 2). The dotted line highlights the one-to-one relation. Middle: same figure for the adopted measure of the $1.6 \mu \mathrm{m}$ $\mathrm{CO}$ band. Bottom: same figure for the adopted measure of the $1.1 \mu \mathrm{m}$ $\mathrm{CN}$ band.

the determination of $T_{\text {eff }}$ from near-IR spectra using the new PHOENIX models.

\subsection{Stellar mass}

We have mentioned in Sect. 3 that the effects of mass on colours and molecular indices, at a given $T_{\text {eff }}$ and $\log (g)$, are small.
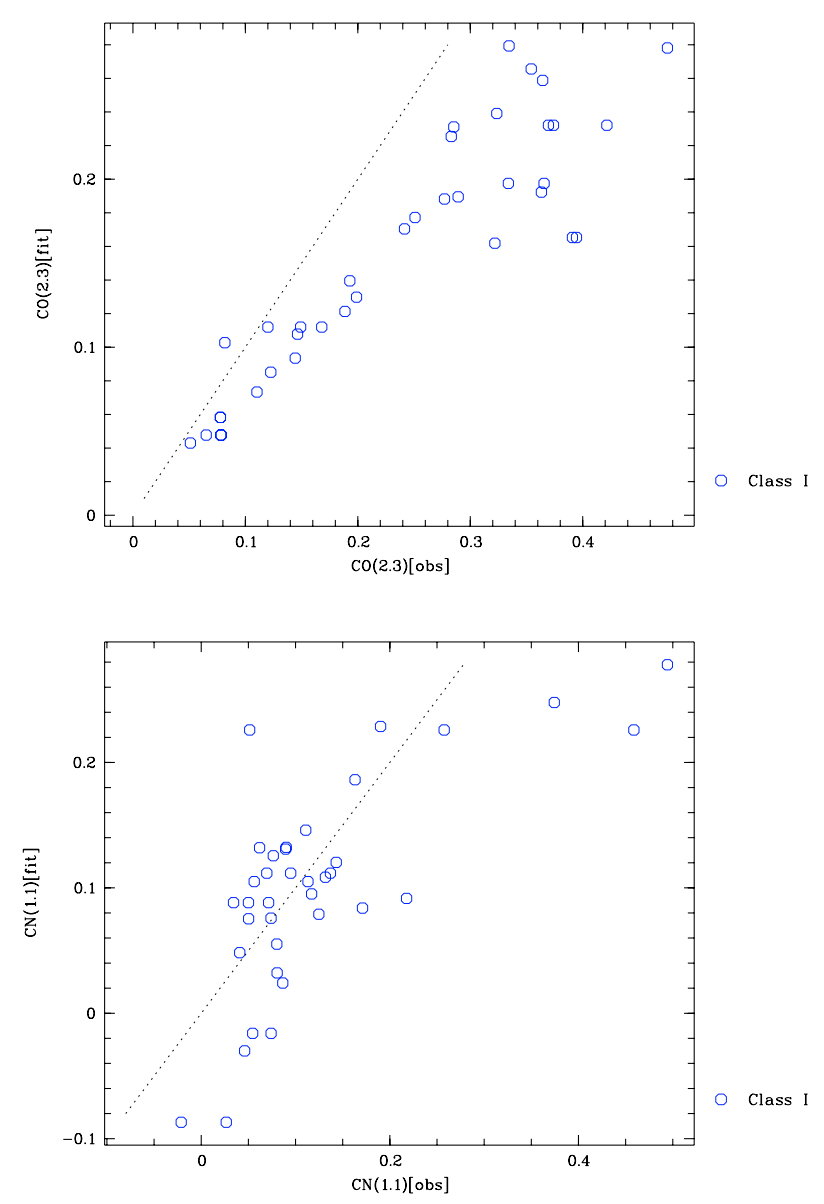

Fig. 19. Same as the top and bottom panels of Fig. 18 but for supergiants only, and using models with RSG-specific abundances.

The comparison between the best fit parameters obtained assuming $1 M_{\odot}$ and $15 M_{\odot}$ nevertheless reveals a trend worth highlighting: for stars with high surface gravities $(\log (g)=2)$, the temperatures obtained with $1 M_{\odot}$ models are systematically lower by $\sim 100 \mathrm{~K}$ than those obtained with $15 \mathrm{M}_{\odot}$ models. This is particularly relevant to giants of class III, but also to the warmer of the bright giants of class II. Unfortunately, we found no systematic differences between the $\chi^{2}$ values obtained with one or the other assumption on mass. Thus, it is not currently possible to determine mass using spectral fits such as those performed in this paper. Mass has to be fixed a priori by other means.

For luminous giants and supergiants, i.e. stars with low gravities, we found no systematic effect of mass on best-fit $T_{\text {eff }}$ or $\log (g)$. The differences in $T_{\text {eff }}$ between the two assumptions are scattered around 0 with typical values of $\pm 100 \mathrm{~K}$ (more in cases where even the best fits are not satisfactory). We note a correlation between the difference in $T_{\text {eff }}$ and the difference in $\log (g)$ : when a change in the assumed mass leads to a rise in the best-fit $T_{\text {eff }}$, it generally also produces a rise of the best-fit value of $\log (g)$.

\section{2. $T_{\text {eff }}$-systematics related to surface abundances: model predictions}

The effects of surface abundance ratios on $T_{\text {eff }}$ estimates (and on derived gravities) are of larger amplitude than those of mass, and we therefore describe them in more detail. They can be 

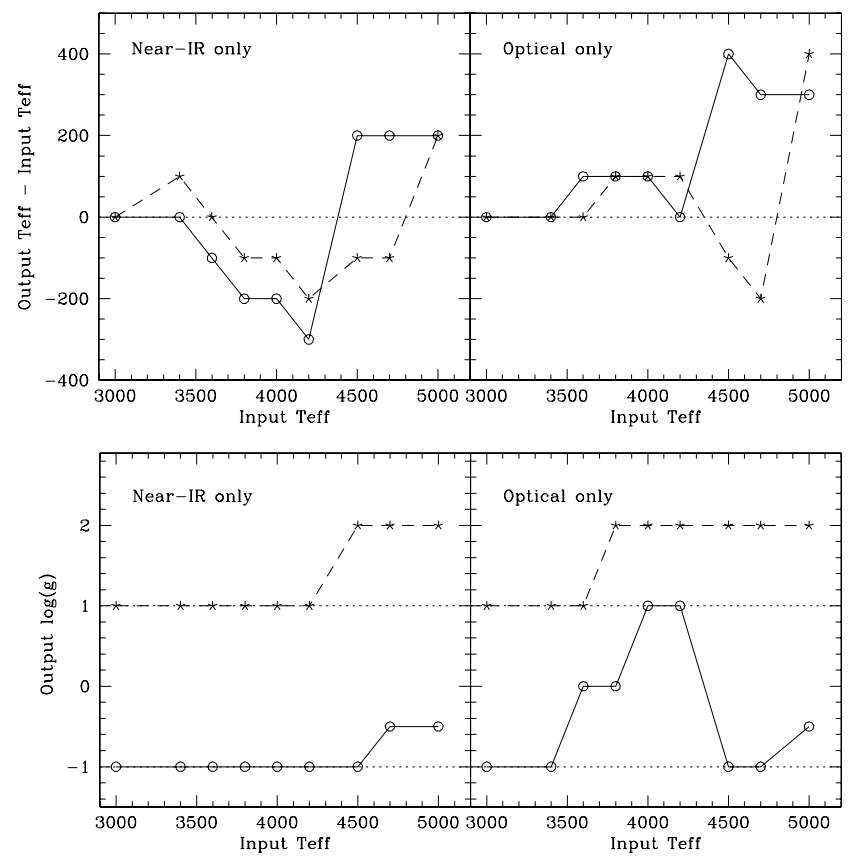

Fig. 20. Effects of the surface abundances on estimates of $T_{\text {eff }}$ and $\log (g)$. The input $T_{\text {eff }}$ and $\log (g)$ refer to solar metallicity models. The output parameters to the values obtained when fitting the solar metallicity spectra with models with RSG-specific abundances, using the procedure described in Sect. 5.1. Solid: input $\log (g)=-1$. Dashed: input $\log (g)=1$.

studied by fitting a sample of solar metallicity models with models with RSG-specific abundances, using the procedure described for fits to observational data. The results depend on the wavelength range adopted in the analysis. They are illustrated in Fig. 20. The amplitude of the effect is of several hundred Kelvin. If we call $\delta T_{\text {eff }}$ the difference between the input and output temperatures (output minus input), we find no simple linear correlation between $\delta T_{\text {eff }}$ and $T_{\text {eff }}$.

The figure based on near-IR data $(0.97-2.4 \mu \mathrm{m}$, with the window and weight functions of Sect.5.1) is tightly related to the behaviour of the near-IR CN bands. Output $T_{\text {eff }}$ values around 4400-4900 K (depending on gravity) are avoided because the $\mathrm{CN}$ bands of those RSG-specific models are too strong. A similar effect is present when optical wavelengths are used $(0.51-0.97 \mu \mathrm{m})$, but it is combined in the low- $T_{\text {eff }}$ range with a variety of effects due to oxides. The difference between the optical and near-IR temperatures is largest between 3500 and $4200 \mathrm{~K}$, where the fluxes below $0.75 \mu \mathrm{m}$ transit rapidly from being almost nil to being large. Eye inspection of the fits shows that the best fit models sometimes deviate wildly from the "data" in the range not included in the fitting procedure, while over the range really used fits are dangerously good. When optical and near-IR spectra are used jointly, compensations occur and the correct Teff is recovered (to within $\pm 100 \mathrm{~K}$ ) below $4200 \mathrm{~K}$. Positive offsets of up to $400 \mathrm{~K}$ however persist above this temperature for all gravities.

The output $\log (g)$ equals the input $\log (g)$ below about $4300 \mathrm{~K}$ when using near-IR data, and is higher by one $\log (g)$-sampling step at higher temperatures. When using optical data, the behaviour depends more strongly on the actual value of the input $\log (g)$. For high gravities, $\delta \log (g)$ is positive (one sampling step) at $T_{\text {eff }}>3600 \mathrm{~K}$. For low gravities, $\delta \log (g)$ is nil at the lowest and highest temperatures, but peaks with a value +2 around $4200 \mathrm{~K}$.

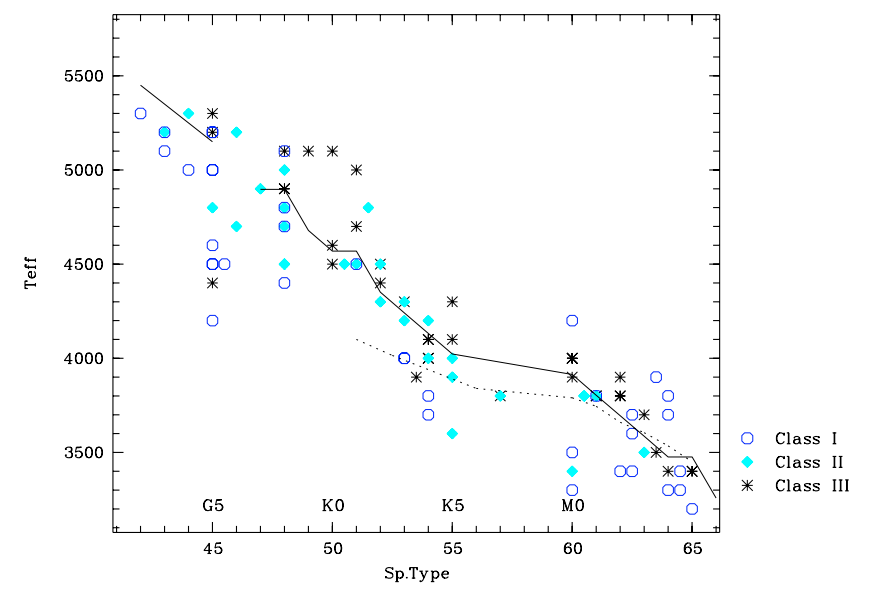

Fig. 21. Effective temperatures derived from fits to near-IR spectra $(\lambda>0.97 \mu \mathrm{m})$, compared with trends in the literature. RSG-specific abundances are used for class I stars, solar abundances for classes II and III. Solid lines: temperature scale for giants, from van Belle et al. (1999) for $T_{\text {eff }}<5000 \mathrm{~K}$ and from Schmidt-Kaler (1982) for $T_{\text {eff }}>5000 \mathrm{~K}$. Dotted line: temperature scale for supergiant stars from Levesque et al. (2005). Default spectral types from the SIMBAD database (operated at CDS, Strasbourg, France) are used for this figure.

We note that corresponding plots can be produced for the "extinction"-correction $A_{\mathrm{V}}$ (which accounts for colour changes in the analysed wavelength ranges reasonably well). The qualitative aspects of the graphs for $\delta A_{\mathrm{V}}$ are similar to those of $\delta T_{\text {eff }}$, with a maximal amplitude of $\pm 0.6 \mathrm{mag}$.

For comparison, we have performed a limited exploration of the effects of metallicity (with solar scaled abundance ratios) on the derived temperatures. Models at $\log \left(Z / Z_{\odot}\right)=-0.3$ were computed for $\log (g)=1$ and -0.5 , and best fits to these were obtained using solar metallicity models. Plots similar to those in Fig. 20 were constructed. The effects of the change in $Z$ on the derived $T_{\text {eff }}$ is notably smaller than those just described for modified abundance ratios. When using optical wavelengths, the trend expected from the well known metallicity-temperature degeneracy is found (lower temperatures are required at lower metallicity to produce similar optical band depths). The offset varies between $100 \mathrm{~K}$ (low $T_{\text {eff }}$ ) and $200 \mathrm{~K}$ (high $T_{\text {eff }}$ ). At near-IR wavelengths, the correct temperatures are recovered unchanged except for a few deviations of $\pm 100 \mathrm{~K}$. In both wavelength ranges, however, gravities higher than input are derived (by one gravity bin). For complementary discussions on metallicity effects, we refer to Kučinskas et al. (2006) and Levesque et al. (2006).

\section{3. $T_{\text {eff }}$ estimates for real stars}

Figure 21 compares the effective temperatures derived in this paper from near-IR spectra with temperature scales from the literature. For giants, the plotted reference scale (below $5000 \mathrm{~K}$ ) is based on angular diameter measurements (van Belle et al. 1999). The number of red supergiants with angular diameter measurements is small. For supergiants, we therefore show the scale recently obtained from fits of MARCS model atmosphere spectra to optical spectra by Levesque et al. (2005). The agreement is good, but the scatter is large.

As a sanity check, we may restrict our data sample to stars with optical spectra and discard near-IR wavelengths $(\lambda>1 \mu \mathrm{m})$ in the fitting procedure. This provides temperatures that are 


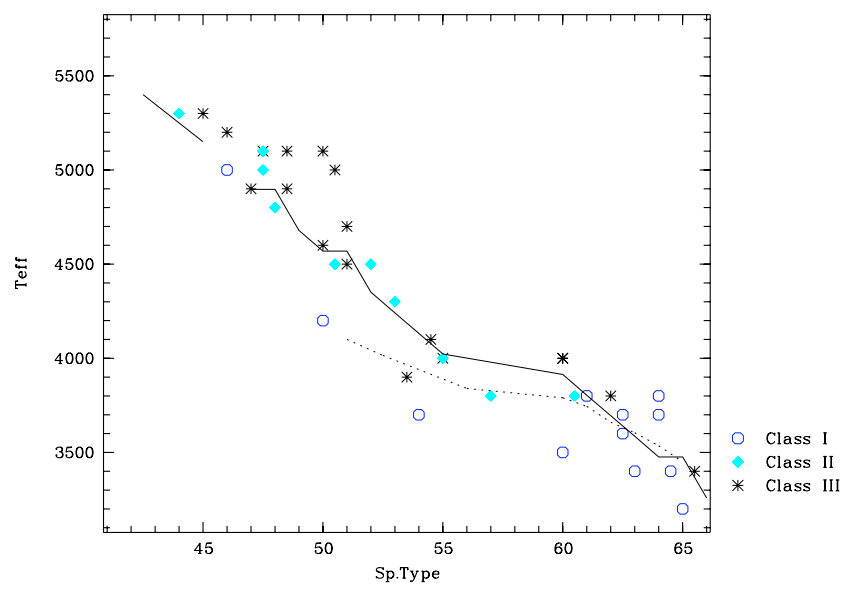

Fig. 22. Same as Fig. 21, but using only spectral types from Keenan \& McNeil (1989) or Buscombe (1998). Using solar abundances moves the G6 supergiant in this figure down $200 \mathrm{~K}$, the $\mathrm{K} 0$ supergiant up $100 \mathrm{~K}$, and the M supergiants above $3600 \mathrm{~K}$ up by 100 to $200 \mathrm{~K}$.

a priori more directly related to spectral types. In addition, we keep only stars with MK spectral types from Keenan \& McNeil (1989) or Buscombe (1998), and with small variability (according to the Simbad database information). Using solar abundance models for direct comparison with the results of Levesque et al. (2005), we find that 8 of the 9 stars in the subsample have estimated temperatures within less than $50 \mathrm{~K}$ of the reference relations. Most of the stars in the subsample are supergiants and all are of type $\mathrm{K} 5$ or later. Thus, in this range of parameters, there is no indication of a systematic difference between the temperatures derived from optical spectra using the new PHOENIX models or the MARCS models of Levesque et al. (2005).

To illustrate what fraction of the scatter in Fig. 21 may be due to spectral classification errors, Fig. 22 reproduces the graph using only MK spectral types from Keenan \& McNeil (1989) or Buscombe (1998), when available. A considerable scatter remains. Some of it is due to the real scatter in the properties of the stars (surface abundances, gravity, unknown variability). For supergiants in particular, and especially at low temperatures, the scatter also reflects the large intrinsic uncertainties associated with the relatively poor quality of the model fits. We expect the spread to shrink once models with a wider range of parameters (surface abundances, micro-turbulent velocities) will have been computed.

We have also examined the diagrams of estimated $T_{\text {eff }}$ vs. spectral type obtained when any available optical data is included in the fitting procedure. They are similar to those described above. Individual stars are moved by up to $200 \mathrm{~K}$, but no systematic trend can be clearly identified (because the stars that move most are also those for which the fits are poorest). Despite the added difficulty of fitting a broader wavelength range, the final dispersion is not significantly enhanced.

\section{Conclusions}

We have presented two grids of PHOENIX models for the spectra of luminous cool stars, one at solar metallicity, the other with RSG-specific surface abundances. We have described the properties of these models and compared them with observations, with a focus on the molecular features found in near-IR spectra at resolution $\lambda / \Delta \lambda \simeq 1000$. At these wavelengths, red giants and supergiants dominate the integrated light of stellar populations. Our main conclusions are the following.

- Models must be computed with a wavelength sampling step of about $0.1 \AA$ in order to reproduce the low resolution nearIR spectra adequately.

- The solar metallicity models provide a very good representation of empirical spectra of giants of class III and of a large fraction of the luminous giants of class II. As expected, RSG-specific abundances are found inadequate for the modelling of the bulk of the giant stars (they are rejected because they provide poorer fits and lead to a zone of avoidance in the derived $T_{\text {eff-distribution). RSG-specific abundances are }}$ favoured for some class II giants, which may have suffered mixing in excess of standard first dredge-up.

- Red supergiant spectra of spectral types G and K, and of luminosity class Ib (sometimes also Iab) can be reproduced reasonably well. Serious disagreements remain in the case of very luminous (Ia and some Iab) and of very cool supergiants (type M). RSG-specific abundances tend to improve the fits to strong $\mathrm{CN}$ bands, although the global effect on the fit quality is not as spectacular as one might have hoped. However, changing the surface abundance ratios has a significant impact on the derived effective temperatures (the effect is larger than that found when moving from $0.5 Z_{\odot}$ to $Z_{\odot}$ ). Therefore, it will remain necessary to account for this effect of stellar evolution in future model grids.

- While it is easy (relatively) to produce good fits to the spectra of either the $J$, or the $H$, or the $K$ band spectra of luminous cool stars, it remains more difficult to reproduce all their optical and near-IR molecular bands simultaneously. As a result, estimated stellar parameters $\left(T_{\text {eff }}, \log (g), A_{\mathrm{V}}\right)$ depend on the spectral range of the analysis. The effects of changes in the surface abundances on these parameters also depend on the wavelengths under study.

- The $T_{\text {eff }}$ scales derived from the comparison of a collection of near-IR stellar spectra $(1-2.4 \mu \mathrm{m})$ with models are generally consistent with previous scales, albeit with considerable scatter. For cool red supergiants, the current uncertainties on individual estimated $T_{\text {eff }}$ values frequently exceed $\pm 100 \mathrm{~K}$.

About $20 \%$ of the analysed red supergiant spectra have such strong $\mathrm{CN}$ bands that they call for models with high microturbulent velocities, and/or even more surface nitrogen than we have considered, and/or for gravities $\operatorname{lower}$ than $\log (g)=-1$. The coolest of these are variable, and variability may contribute to the building of an extended atmosphere with low effective gravities. Large micro-turbulent velocities have been derived for a number of red supergiants in the past, and our first calculations confirm that increasing this parameter will help reproducing the spectra of type Ia supergiants. In particular, a better agreement with observations is expected for the ratio between the first and second overtone $\mathrm{CO}$ band strengths. A grid of models is currently being calculated. Somewhat higher nitrogen abundances than we have explored are expected to exist in nature, for instance when stellar rotation increases internal mixing. Because low resolution near-IR spectra of red supergiants are relatively easy to acquire, their comparison with models at the specific abundances predicted by stellar tracks with rotation will provide interesting tests of stellar evolution theory. Considering stars with lower gravities is a more challenging modelling task, as they will develop strong winds. In addition, the winds may be loaded with dust. Since winds are a well known empirical property of many red supergiants, the development of models that include winds is a necessity of the future. 
Acknowledgements. PHH was supported in part by the Pôle Scientifique de Modélisation Numérique at ENS-Lyon and by Université Louis Pasteur at Strasbourg Observatory. Some of the calculations presented here were performed at the Höchstleistungs Rechenzentrum Nord (HLRN), and at the National Energy Research Supercomputer Center (NERSC), supported by the U.S. DOE, and at the computer clusters of the Hamburger Sternwarte, supported by the DFG and the State of Hamburg. We thank all these institutions for a generous allocation of computer time. We thank C. Charbonnel for insightful discussions of aspects of this work. This research has made use of the SIMBAD database and the VIZIER service, operated at CDS, Strasbourg, France. It uses data (in preparation for publication) acquired using the NASA Infrared Telescope Facility, Hawaii, USA, and the $2.3 \mathrm{~m}$ Telescope of the Australian National University, Siding Spring, Australia.

\section{References}

Anders, E., \& Grevesse, N. 1989, Geochim. Cosmochim. Acta, 53, 197 Asplund, M., Grevesse, N., \& Sauval, A. J. 2006, in Cosmic Abundances as Records of Stellar Evolution and Nucleosynthesis, ed. T. G. Barnes, III, \& F. N. Bash, ASP Conf. Ser., 336, 25

Baldwin, J. R., Frogel, J. A., \& Persson, S. E. 1973, ApJ, 184, 427

Becker, S. A., \& Iben, I., Jr. 1979, ApJ, 232, 831

Beer, R., Hutchison, R. B., Norton, R. H., \& Lambert, D. L. 1972, ApJ, 172, 89

Bessell, M. S., \& Brett, J. M. 1988, PASP, 100, 1134

Bessell, M. S., Brett, J. M., Scholz, M., \& Wood, P. R. 1989, A\&AS, 77, 1

Bessell, M. S., Brett, J. M., Scholz, M., \& Wood, P. R. 1991, A\&AS, 89, 335

Boothroyd, A. I., \& Sackmann, I.-J. 1999, ApJ, 510, 232

Bressan, A., Fagotto, F., Bertelli, G., \& Choisi, C. 1993, A\&AS, 100, 647

Buscombe 1998, Illinois catalogue of MK spectral classifications (version 13),

Northwestern Univ. Evanston, Illinois.

Cardelli, J. A., Clayton, G. C., \& Mathis, J. S. 1989, ApJ, 345, 245

Charbonnel, C. 1994, A\&A, 282, 811

Charbonnel, C., \& do Nascimento, J. D., Jr. 1998, A\&A, 336, 915

Charbonnel, C., Meynet, G., Maeder, A., \& Schaerer, D. 1996, A\&AS, 115, 339

Decin, L., Shkedy, Z., Molenberghs, G., Aerts, M., \& Aerts, C. 2004, A\&A, 421, 281

Frogel, J. A., Persson, S. E., Aaronson, M., \& Matthews, K. 1978, ApJ, 220, 75

Girardi, L., Bressan, A., Bertelli, G., \& Chiosi, C. 2000, A\&AS, 141, 371

Goorvitch, D., \& Chackerian, C. 1994a, ApJS, 91, 483

Goorvitch, D., \& Chackerian, C. 1994b, ApJS, 92, 311

Grevesse, N. 1991, in Evolution of Stars: the Photospheric Abundance Connection, ed. G. Michaud, \& A. V. Tutukov (Dordrecht: Kluwer), IAU Symp., 145, 63
Grevesse, N., \& Noels, A. 1993, in Origin and Evolution of the Elements, ed. N. Prantos, E. Vangioni-Flam, \& M. Cassé (Cambridge Univ. Press), 14

Iben, I., Jr. 1964, ApJ, 140, 1631

Iben, I. 1966, ApJ, 143, 516

Keenan, P. C., \& McNeil, R. C. 1989, ApJSS, 71, 245

Kučinskas, A., Hauschildt, P. H., Ludwig, H.-G., et al. 2005, A\&A, 442, 281

Kučinskas, A., Hauschildt, P. H., Brott, I., et al. 2006, A\&A, 452, 1021

Lançon, A., \& Wood, P. R. 2000, A\&AS, 146, 217

Lançon, A., Smith, L. J., Gallagher, J. S., et al. 2003, in Extragalactic Globular Clusters and their Host Galaxies (IAU JD 6, ed. T. Bridges), Highlights of Astronomy 13, ed. O. Engvold, in press

Lançon, A., Wood, P. R., Ladjal, D., et al. 2007, in preparation

Levesque, E. M., Massey, P., Olsen, K. A. G., et al. 2005, ApJ, 628, 973

Levesque, E. M., Massey, P., Olsen, K. A. G., et al. 2006, ApJ, 645, 1102

Loidl, R., Lançon, A., \& Jorgensen, U. G. 2001, A\&A, 371, 1065

Maeder, A. 1981, A\&A, 101, 385

Maeder, A., \& Meynet, G. 2001, A\&A, 373, 555

Martins, L. P., González Delgado, R. M., Leitherer, C., Cerviño, M., \& Hauschildt, P. H. 2005, MNRAS, 358, 49

McGregor, P. J. 1987, ApJ, 312, 195

McWilliam, A., \& Lambert, D. L. 1984, PASP, 96, 882

Meynet, G., \& Maeder, A. 2000, A\&A, 361, 101

Meynet, G., \& Maeder, A. 2003, A\&A, 404, 975

Meynet, G., Maeder, A., Schaller, G., Schaerer, D., \& Charbonnel, C. 1994, A\&AS, 103, 97

Origlia, L., Moorwood, A. F. M., \& Oliva, E. 1993, A\&A, 280, 536

Origlia, L., Ferraro, F. R., Fusi Pecci, F., \& Oliva, E. 1997, A\&A, 321, 859

Schaller, G., Schaerer, D., Meynet, G., \& Maeder, A. 1992, A\&AS, 96, 269

Schmidt-Kaler 1982, in Astrophysical Data: Planets \& Stars, 1992, ed. N. Lang (New York: Springer)

Schwenke, D. W. 1998, in Chemistry \& Physics of Molecules and Grains in Space. Faraday Discussions No. 109 (London: Royal Soc. of Chemistry), 321

Smith, V. V., \& Lambert, D. L. 1985, ApJ, 294, 326

Trundle, C., \& Lennon, D. J. 2005, A\&A, 434, 677

Tsuji, T. 1976, PASJ, 28, 543

van Belle, G. T., Lane, B. F., Thompson, R. R., et al. 1999, AJ, 117, 521

Vanhollebeke, E., Blommaert, J. A. D. L., Schultheis, M., Aringer, B., \& Lançon, A. 2006, A\&A, 455, 645

White, N. M., \& Wing, R. F. 1978, ApJ, 222, 209

Wing, R. F., \& Spinrad, H. 1970, ApJ, 159, 973 